\title{
Effect of Wind Direction and Incidence Angle on Polarimetric SAR Observations of Slicked and Unslicked Sea Surfaces
}

\author{
Stine Skrunes ${ }^{\mathrm{a}, *}$, Camilla Brekke ${ }^{\mathrm{a}}$, Cathleen E. Jones ${ }^{\mathrm{b}}$, Martine M. Espeseth $^{\mathrm{a}}$, \\ Benjamin Holt ${ }^{\mathrm{b}}$ \\ ${ }^{a}$ Department of Physics and Technology, UiT The Arctic University of Norway, Tromsø, \\ Norway \\ ${ }^{b}$ Jet Propulsion Laboratory, California Institute of Technology, Pasadena, CA, USA
}

\begin{abstract}
The objective of this paper is to investigate the dependency of oil spill observations in polarimetric SAR data on imaging geometry, i.e., on incidence angle and look direction relative to the wind. The study is based on quad-polarization data acquired by the Uninhabited Aerial Vehicle Synthetic Aperture Radar over experimental oil slicks under relatively high winds of 10-12 m/s over an 8-hour period. The data is collected over a wide range of incidence angles and alternates between looking upwind (UW) and downwind (DW). The unique time series enables a detailed study of the behavior of multipolarization parameters over clean sea and oil slicks under varying imaging geometry to be carried out for the first time. For clean sea backscatter, our findings are in agreement with previous studies, showing decreasing backscatter as the incidence angle increases and from UW to DW, with the highest sensitivity in the HH channel. We also find similar variations in oil covered areas. The results suggest that the oil slick backscatter is slightly more sensitive to the relative wind direction than the clean sea, and higher oil-sea damping ratios are found in DW than in UW cases, particularly in the $\mathrm{HH}$ channel. All multipolarization features investigated have some degree of dependency on imaging geometry. The
\end{abstract}

\footnotetext{
* Corresponding author

Email address: stine.skrunes@uit.no (Stine Skrunes)
}

Preprint submitted to Remote Sensing of Environment

April 24, 2018 
lowest sensitivities are found in the magnitude of the copolarization correlation coefficient, the standard deviation of the copolarized phase difference, the polarization difference, the mean scattering angle and the entropy. Several features clearly change behavior when the signal approaches the sensor noise floor, and we find that the measurements and derived parameters may be affected at even higher signal-to-noise ratio (SNR) levels than previously proposed, i.e., closer to $7-9 \mathrm{~dB}$ above the sensor noise floor. Overall, the polarization difference is clearly identified as the most interesting parameter for oil spill observation, producing high oil-sea contrast in addition to low sensitivity to imaging geometry. The results show that both the relative wind direction and the incidence angle, in combination with the SNR, should be taken into account when developing operational methods based on multipolarization SAR data.

Keywords: oil spill, synthetic aperture radar (SAR), polarimetry, imaging geometry, incidence angle, wind direction, SNR, ocean scattering

\section{Introduction}

Synthetic Aperture Radar (SAR) is a well-established remote sensing tool for detection of illegal and accidental oil spills, and can be useful in clean-up operations during oil spill events. Currently, low resolution single-polarization SAR images are used in daily operational oil spill services, but the application of multipolarization SAR for improving oil spill detection and characterization have been extensively investigated over the last decade (see, e.g., Nunziata et al. 8 (2008); Migliaccio et al. (2009a); Minchew et al. (2012); Skrunes et al. (2014)). 9 The measurements and derived parameters are affected by a number of factors related to SAR sensor configuration and environmental conditions, which can complicate the data analysis and interpretation (see, e.g., Skrunes et al. (2015a, 2016a)). Hence, before multipolarization data can be used operationally, better knowledge of these effects is needed to develop accurate and reliable methods with a large and known range of validity. It is also of interest to identify features with good detection capabilites as well as low dependency on sensor and 
environmental factors. This paper is a first attempt at a detailed investigation of these questions, made possible through use of multiple images acquired in close succession using an airborne SAR.

The objective of this work is to investigate how oil spill observations using polarimetric SAR are affected by the sensor incidence angle and the look direction relative to the wind (herein referred to in combination as imaging geometry). The effect on both the individual polarization channels and on multipolarization features are investigated for clean sea and for oil covered surfaces. Although the dependency of clean sea backscatter on imaging geometry is well described in the literature, few studies have looked at the effects on oil covered regions and their detectability, and on multipolarization parameters. This study provides new insight into these effects, by evaluating the features behavior for both changing incidence angle and relative wind direction, also enabling identification of parameters with less sensitivity to these factors. The study is based on data acquired over experimental oil slicks in the North Sea by the National Aeronautics and Space Administration (NASA) Uninhabited Aerial Vehicle Synthetic Aperture Radar (UAVSAR), which is an airborne L-band quad-polarization SAR instrument. The unique time series makes it possible to do a detailed investigation of the imaging geometry effects on polarimetric SAR data over slicked and unslicked sea surfaces for the first time.

The paper is organized as follows. Background information on ocean radar backscatter and application of polarimetric SAR for oil spill observation is given in Section 2, and the data set is described in Section 3. The results are presented in Sections 4 and 5, and Section 6 concludes the paper.

\section{Background}

The following subsections contain some background information on ocean radar backscatter and the effect of imaging geometry on polarimetric SAR measurements, particularly from the oil spill observation perspective. 


\subsection{Ocean Backscatter}

The SAR backscatter from ocean surfaces depends on a number of factors related to sensor properties and surface characteristics. The general behavior of the ocean backscatter is well known, see, e.g., Ulaby et al. (1986); Donelan and Pierson (1987), and a vast amount of research has been done on the relation between SAR backscatter and wind conditions and imaging geometry (see, e.g., Dagestad et al. (2012) and references therein). For incidence angles above ca. $30^{\circ}$, the largest backscatter is found in the $\mathrm{VV}$ (vertical transmit and receive) channel, somewhat lower values in the $\mathrm{HH}$ (horizontal transmit and receive) channel, and the lowest signal in the HV (horizontal transmit and vertical receive) channel. The backscatter decreases when the incidence angle increases, with the steepest slope in the $\mathrm{HH}$ channel; increases with wind speed; and varies with the radar look direction relative to the wind direction (Ulaby et al., 1986). The latter dependency is specified as a function of the azimuth angle, $\psi$, defined as the angle between the radar look direction and the upwind direction, i.e., $\psi=0^{\circ}$ and $\psi=180^{\circ}$ denotes upwind (UW) and downwind (DW), respectively. In general, the backscatter maximum is found in UW, a smaller signal in DW, and minima when the sensor is looking perpendicular to the wind direction, i.e., crosswind $(\mathrm{CW})$. The larger maxima in UW can be related to presence of foam and enhanced growth of short capillary-gravity waves on the downwind face of longer waves (Zhou et al., 2017). The backscatter difference between wind directions is larger in the $\mathrm{HH}$ channel than in VV (Ulaby et al., 1986).

Although most studies of ocean backscatter have been based on C-band SAR data, these general characteristics have been observed also for L-band in, e.g., Isoguchi and Shimada (2009); Yueh et al. (2010, 2013, 2014); Zhou et al. (2017). At wind speeds comparable to the conditions in the data set investigated in this paper (ca $12 \mathrm{~m} / \mathrm{s}$ ), the highest $\mathrm{HH}$ and $\mathrm{VV}$ backscatter were found in UW, slightly lower in DW, and lowest in CW for incidence angles between $29^{\circ}$ - 46 ${ }^{\circ}$. Isoguchi and Shimada (2009) found that DW backscatter exceeds UW backscatter for small $\theta$ below about $25^{\circ}$. The difference between UW and DW 
backscatter was lower in VV than in $\mathrm{HH}$. Differences of about $0.5 \mathrm{~dB}$ and $2 \mathrm{~dB}$ were found in Yueh et al. (2013) for VV and HH, respectively. The sensitivity of the ocean backscatter to wind direction, especially the UW-DW difference, was found to increase with wind speed and incidence angle in Isoguchi and Shimada (2009); Yueh et al. (2010, 2013, 2014); Zhou et al. (2017). However, at wind speeds above $20 \mathrm{~m} / \mathrm{s}$, Yueh et al. (2013) found a reduction in the $\psi$ dependency, which the authors suggested could be due to an increasing presence of breaking waves and sea foam that have more isotropic scattering signatures than wind-generated waves. Most studies have focused on the wind dependency of copolarization channels. However, some cross-polarization data are included in Yueh et al. (2010) and Yueh et al. (2014). Yueh et al. (2010) found similar $\psi$-dependency in all polarization channels, with peaks in UW and DW and dips in $\mathrm{CW}$ for $\theta=45^{\circ}$, but the UW-DW difference appeared to be smaller in the HV channel compared to in copolarization data. In Yueh et al. (2014), higher backscatter in DW than UW was observed for wind speeds above $12 \mathrm{~m} / \mathrm{s}$ at $\theta$ of $29^{\circ}$ and partly at $38^{\circ}$, which is the opposite of the general behavior in the copolarization channels. This was not observed at $46^{\circ}$.

The sensitivity to wind conditions varies between the different radar frequencies, as described in, e.g., Donelan and Pierson (1987). Isoguchi and Shimada (2009) found comparable wind sensitivity in C- and L-band at wind speeds $>10 \mathrm{~m} / \mathrm{s}$ and small $\theta$, whereas a lower wind sensitivity was found in L-band than in C-band for moderate wind and large $\theta$. In Unal et al. (1991), larger variation between UW and DW was found in C-band compared to L-band at $10 \mathrm{~m} / \mathrm{s}$ wind.

In the absence of long waves, the ocean backscatter within typical SAR incidence angles $\left(\sim 18^{\circ}-50^{\circ}\right)$ is dominated by Bragg scattering, i.e., waves with wavelength $\lambda_{B}=\left(n \lambda_{r}\right) /(2 \sin \theta)$, where $\lambda_{r}$ is the radar wavelength and $n=1,2, \ldots$ is the order of resonance ( $n=1$ produces the dominant return) (Valenzuela, 1978; Ulaby et al., 1986, p. 842). For the UAVSAR instrument with a frequency of $1.26 \mathrm{GHz}, \lambda_{B}$ varies from $13 \mathrm{~cm}\left(\right.$ at $\left.\theta=67^{\circ}\right)$ to $32 \mathrm{~cm}$ (at $\theta=22^{\circ}$ ). The two-scale approximation is a more representative scattering 
model than the Bragg model, as it also takes into account the effects of longer ocean waves on the local incidence angle and roughness through tilt and hydrodynamic modulations (Holt, 2004; Vachon et al., 2004). The HH channel is more sensitive to changes in the local incidence angle than VV, and hence more affected by the tilt caused by larger waves (Thompson, 2004), and also more sensitive to whitecapping and wave steepness which can cause UW-DW difference (Donelan and Pierson, 1987). More recent scattering models describe the radar return as a sum of a polarized Bragg scatter component and a non-polarized component (Kudryavtsev et al., 2003; Mouche et al., 2006; Kudryavtsev et al., 2013). The nonpolarized component has been shown to account for most of the differences observed between UW and DW backscatter (i.e., the so-called UWDW asymmetry) (Mouche et al., 2006). This nonpolarized scattering can be specular reflections due to enhanced roughness or larger slopes of steep waves, e.g., associated with breaking waves. The relative contribution of the nonpolarized component increases from DW to UW, from low to high wind speed, from VV to HH and with incidence angle (Mouche et al., 2006). The latter may also be related to a closer proximity to noise floor at higher $\theta$. Breaking waves were also included in the recent scattering model in Plant and Irisov (2017), and were found to produce UW-DW asymmetry mainly at incidence angles above $45^{\circ}$ and in the $\mathrm{HH}$ channel. An additional term describing specular reflection from steep slopes can be included in the scattering models, in particular for describing the scattering at very low incidence angles, when applicable (Ulaby et al., 1986; Mouche et al., 2006).

In Section 4.2, the L-band ocean backscatter in the UAVSAR time series here investigated will be discussed and compared to these previous studies.

\subsection{Oil Spill Detection and Imaging Geometry}

Although the effect of imaging geometry on the characteristics of ocean backscatter in polarimetric SAR is relatively well described in the literature, few studies have been done looking at these effects for slick-covered water, including effects on the multipolarization parameters recently applied in the oil spill lit- 
erature. The most relevant study is Minchew et al. (2012), in which UAVSAR data acquired over the Deepwater Horizon oil spill, covering incidence angles from $22^{\circ}-65^{\circ}$, were investigated, although the geometry aspect was not the focus of the paper. For the two UAVSAR scenes analysed, a general increase in oil-sea contrast (damping ratio) with incidence angle was observed for data well above the sensor noise floor. At high incidence angles, where the signal was approaching the noise floor in $\mathrm{HH}$ and $\mathrm{HV}$, the damping ratio started to decrease (Minchew et al., 2012). Increasing damping ratio with incidence angle has also been found in simulation studies (Pinel et al., 2014).

As the backscatter decreases with increasing incidence angle, the signal approaches the sensor noise floor, i.e., the noise equivalent sigma zero (NESZ). In Minchew et al. (2012), backscatter values lower than $6 \mathrm{~dB}$ above the noise floor were considered corrupted by the sensor noise and unsuited for analysis of scattering properties. If the backscatter in one or several channels is close to the NESZ, an apparent randomness will be induced that is not representative of the actual physical properties of the surface (Minchew et al., 2012). Hence, a low signal-to-noise ratio (SNR) can also affect multipolarization features and their interpretation. The proximity of the measurements to the given sensor noise floor should always be considered in oil spill analysis, particularly if radar-dark surface characterization is the objective. As the SNR generally decreases with increasing $\theta$ for satellite SARs, the proximity to the noise floor must also be taken into consideration when discussing variations with incidence angle. For many SAR sensors, particularly spaceborne sensors, the noise can affect the measurements even at relatively low incidence angles due to a higher NESZ than airborne SARs.

When it comes to the radar look direction relative to the wind, some early studies found oil spill damping ratios to be independent of this factor using data from the spaceborne SIR-C/X-SAR ( Gade et al., 1998) and airborne HELISCAT scatterometer (Wismann et al., 1998). On the other hand, Minchew et al. (2012) observed differences in damping ratios between scenes of opposite look direction, which was suggested to be due to the difference in wind direction and its effect 


\subsection{Oil Spill Observation in Polarimetric SAR}

A full-polarimetric SAR system measures all four combinations of linear transmit and receive polarizations, i.e., the full scattering matrix $\mathbf{S}$ :

$$
\mathbf{S}=\left[\begin{array}{ll}
S_{H H} & S_{V H} \\
S_{H V} & S_{V V}
\end{array}\right]=\left[\begin{array}{ll}
\left|S_{H H}\right| e^{j \phi_{H}} & \left|S_{V H}\right| e^{j \phi_{V H}} \\
\left|S_{H V}\right| e^{j \phi_{H V}} & \left|S_{V V}\right| e^{j \phi_{V V}}
\end{array}\right]
$$

where $\left|S_{X Y}\right|$ and $\phi_{X Y}$ denote the amplitude and phase of the measured complex scattering coefficients, and the first and second subscript refer to transmit and receive polarization, respectively. Assuming reciprocity, $S_{H V}=S_{V H}$, the Pauli scattering vector, $\mathbf{k}$, can be extracted from the scattering matrix as:

$$
\mathbf{k}=\frac{1}{\sqrt{2}}\left[\begin{array}{lll}
S_{H H}+S_{V V} & S_{H H}-S_{V V} & 2 S_{H V}
\end{array}\right]^{T}
$$

where the superscript $T$ denotes the transpose operator (Lee and Pottier, 2009). From $\mathbf{k}$, the $3 \times 3$ coherency matrix $\mathbf{T}$ can be computed:

$$
\mathbf{T}=\frac{1}{L} \sum_{n=1}^{L} \mathbf{k}_{n} \mathbf{k}_{n}^{* T}
$$

where $\mathbf{k}_{n}$ is the single look complex (SLC) measurement corresponding to pixel number $n, L$ is the number of samples included in the averaging and the superindex $*$ denotes complex conjugate. The resulting matrix is:

$$
\begin{aligned}
& \mathbf{T}= \\
& \frac{1}{2}\left[\begin{array}{lll}
\left\langle\left|S_{H H}+S_{V V}\right|^{2}\right\rangle & \left\langle\left(S_{H H}+S_{V V}\right)\left(S_{H H}-S_{V V}\right)^{*}\right\rangle & 2\left\langle\left(S_{H H}+S_{V V}\right) S_{H V}^{*}\right\rangle \\
\left\langle\left(S_{H H}-S_{V V}\right)\left(S_{H H}+S_{V V}\right)^{*}\right\rangle & \left\langle\left|S_{H H}-S_{V V}\right|^{2}\right\rangle & 2\left\langle\left(S_{H H}-S_{V V}\right) S_{H V}^{*}\right\rangle \\
2\left\langle S_{H V}\left(S_{H H}+S_{V V}\right)^{*}\right\rangle & 2\left\langle S_{H V}\left(S_{H H}-S_{V V}\right)^{*}\right\rangle & 4\left\langle\left|S_{H V}\right|^{2}\right\rangle
\end{array}\right],
\end{aligned}
$$

where $\langle\cdot\rangle$ indicates ensemble averaging (Lee and Pottier, 2009).

Polarimetry is a powerful tool for SAR data analysis, and can be used to infer information about the physical properties of the observed areas, including surface roughness and dielectric properties. Over the last decade, multipolarization SAR data have been extensively investigated to evaluate its potential for improved oil spill detection and characterization. Some studies find promising 
results for separating actual oil spills from natural phenomena such as biogenic slicks (see, e.g., Nunziata et al. (2008); Migliaccio et al. (2009a); Kudryavtsev et al. (2013); Skrunes et al. (2014)), and for estimation of oil content in emulsions (see, e.g., Minchew et al. (2012)). Although quad-polarization data are not used operationally today, mainly due to availability and the lower spatial coverage compared to single-polarization modes, these data types may be increasingly used in the future. However, in order to use multipolarization features more operationally for ocean monitoring or in a clean-up situation, additional information about how they are affected by various factors such as SAR sensor configuration and environmental conditions, are needed. In this study, the dependency on two of these factors, i.e., the incidence angle and the relative wind direction, are evaluated for 12 multipolarization features that have previously been used in oil spill studies in, e.g., Migliaccio et al. (2007); Nunziata et al. (2008); Migliaccio et al. (2009b, 2011a); Velotto et al. (2011); Zhang et al. (2011); Liu et al. (2011); Minchew et al. (2012); Kudryavtsev et al. (2013); Skrunes et al. (2014, 2015b); Brekke et al. (2016); Latini et al. (2016); Singha et al. (2016); Hansen et al. (2016); Skrunes et al. (2016a); Espeseth et al. (2017). These are defined in Table 1. Each feature is here calculated from the UAVSAR SLC data using a sliding window of size $15 \times 61$ pixels (similar to what is used in Jones et al. (2016a); Espeseth et al. (2017)). In Espeseth et al. (2017), the two-scale Bragg scatter model (see, e.g., Salberg et al. (2014)) was applied to categorize multipolarization features based on their dependency on various factors. The category to which the different features belong is indicated in Table 1. Category I contains features that depend on large- and small-scale roughness, $\theta$, and dielectric constant, whereas the features in category II only depend on large-scale roughness, $\theta$, and dielectric constant. These category II features are ratio-based parameters where the wave spectrum cancels out. Note that as the categorization is based on the two-scale Bragg model, the classification of features is not valid outside the validity range of this model, e.g., at very low incidence angles where contributions from specular reflections may dominate. Further details on the categorization and its relation to the two-scale Bragg 
model equations are found in Espeseth et al. (2017).

The Span and the Geometric intensity $(\mu)$ are both measures of the combined intensity in HH, VV and HV channels. In Skrunes et al. (2015b), the $\mu$ based on $\mathrm{HH}$ and $\mathrm{VV}$ intensity (HV was excluded due to low SNR) was found to be useful for discriminating between oil spills and clean sea and between mineral oil and plant oil. The Copolarization power ratio $\left(\gamma_{C O}\right)$ has been used to detect changes in the dielectric constant due to presence of thick oil spill in Minchew et al. (2012). The Polarization difference $(P D)$ is controlled by surface roughness caused by wave components that are close to the Bragg wavenumber, and should reflect near-surface wind variability and reveal the presence of slicks (Kudryavtsev et al., 2013). It's been found to have very good oil detection capabilities in, e.g., Kudryavtsev et al. (2013); Skrunes et al. (2015b). The Standard deviation of the copolarized phase difference $\left(\sigma_{\phi C O}\right)$ measures the degree of correlation between $S_{H H}$ and $S_{V V}$. It has been found to emphasize the presence of oil slicks as areas of decreased correlation, while deemphasizing the presence of look-alikes in, e.g., Migliaccio et al. (2009a), where the difference was related to a change in scattering mechanisms. Decorrelation effects have also been detected using the Magnitude of the copolarization correlation coefficient $\left(\rho_{C O}\right)$ and the Real part of the copolarization cross product $\left(r_{C O}\right)$. The latter have been found to give promising results for oil vs. look-alike discrimination in, e.g., Nunziata et al. (2008); Skrunes et al. (2014). In Brekke et al. (2017), the Standard deviation of the copolarization cross product magnitude $\left(\sigma_{z C O}\right)$ was included for a more complete description of the correlation properties, and found to produce interesting internal zoning in an oil slick, possibly correlated with dispersion activities. The final four features in Table 1 are related to the $H / A / \bar{\alpha}$ decomposition described in Cloude and Pottier (1997). The Entropy $(H)$ is a measure of the randomness of the scattering process, and takes values between 0 (one dominating scattering mechanism) and 1 (random scattering). The Mean scattering angle $(\bar{\alpha})$ indicates the type of scattering that is dominating, and varies from $0^{\circ}$ to $90^{\circ}$. Low $\bar{\alpha}$ indicates surface scattering, intermediate $\bar{\alpha}$ volume scattering, and high $\bar{\alpha}$ double bounce scattering. Bragg 


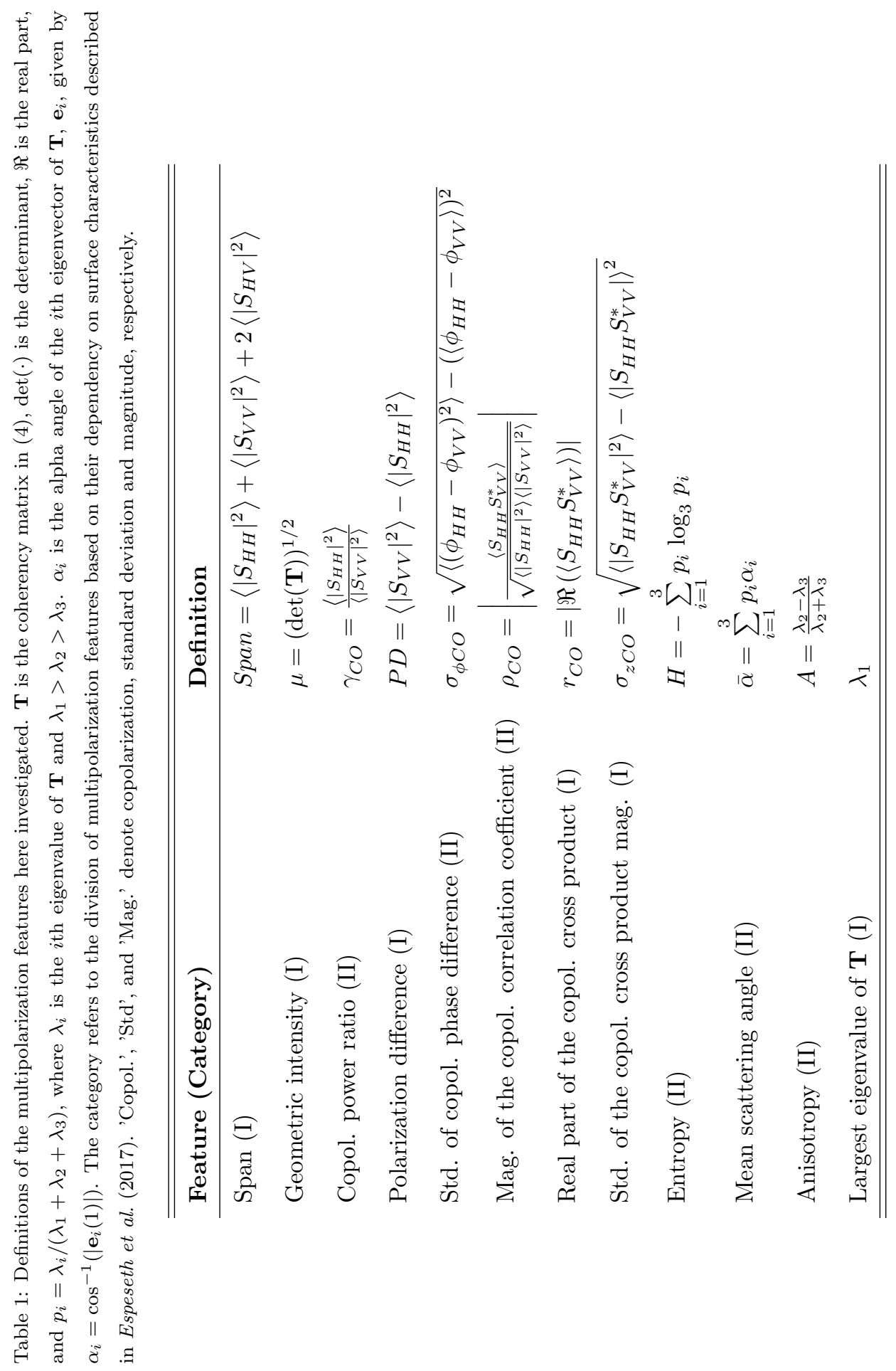


scattering is traditionally defined in the $H-\bar{\alpha}$ plane as the region with $H<0.5$ and $\bar{\alpha}<42.5^{\circ}$ (Lee and Pottier, 2009). The Anisotropy $(A)$ is a measure of the relative importance of the second and third eigenvalues of T. $A$ is only useful when the $H$ is high, $H>0.7$, otherwise $\lambda_{2}$ and $\lambda_{3}$ are highly affected by noise (Lee and Pottier, 2009). Several studies have applied the $H / A / \bar{\alpha}$ decomposition for oil spill observation, and a discrimination between oil spills and look-alikes based on a change in scattering mechanism from Bragg scatter to more random scattering has been proposed (see, e.g., Migliaccio et al. (2007, 2011b); Tian et al. (2010)). However, low SNR can also alter the parameters in this direction (Minchew et al., 2012; Alpers et al., 2017), causing some uncertainty on the applicability of these features. The largest eigenvalue of $\mathbf{T}, \lambda_{1}$, has been found to be a relatively robust oil detection parameter, with low sensitivity to sensor noise in, e.g., Minchew et al. (2012). The application of multipolarization SAR for oil spill observation is further described in, e.g., Skrunes et al. (2014, 2016a) and references therein.

It is noted that the parameters defined in Table 1 are partly correlated, see, e.g., Singha et al. (2016). However, we here discuss each parameter individually to evaluate each feature's behavior with changing imaging geometry, independently of between-feature correlations.

Although multipolarization parameters have been investigated for oil spill observation in many studies, the effect of imaging geometry on their values, interpretation and performance have had fewer studies. In Minchew et al. (2012), some multipolarization features were analysed for UAVSAR data, and their variation with incidence angle for both clean sea and an oil spill were plotted. For the $\gamma_{C O}$, the results in Minchew et al. (2012) showed decreasing values with increasing $\theta$ for both oil and clean sea. At the highest $\theta$, where the HH backscatter was approaching the noise floor, the values started to increase. The $H$ and $\bar{\alpha}$ were both found to increase with $\theta$, and to indicate Bragg scatter for both clean sea and oil slicks for all measurements above the SNR threshold defined by the authors. At high incidence angles, the $H$ for oil-covered areas exceeded that of clean sea and sharply increased, which the authors in Minchew et al. (2012) 
related to the noise having a significant contribution on the signal. The $A$ was found to be incidence angle dependent with values decreasing with increasing $\theta$ for low-intermediate angles before reaching a minimum. The dependency of $\gamma_{C O}$ and $P D$ on imaging geometry have been thoroughly investigated for Cband SAR and clean sea in, e.g., Mouche et al. (2005, 2006), for the purpose of scattering model development. The $\gamma_{C O}$ was found to decrease with increasing incidence angle from $\gamma_{C O} \sim 1$ at $\theta<20^{\circ}$, and from CW to UW and from UW to DW. The UW-DW difference in $\gamma_{C O}$ was negligible below $\theta \sim 30^{\circ}$, but increased with $\theta$ above this value (Mouche et al., 2005). The authors in Mouche et al. (2006) found that the variations in $\gamma_{C O}$ with $\theta$ and $\psi$ could not be explained using only the Bragg model, and that the nonpolarized component, e.g., due to breaking waves was required to obtain a match between the model and observations (see Section 2.1). In $P D$ on the other hand, the nonpolarized component is removed, and only the Bragg components remain. In Mouche et al. (2006), decreasing values of $P D$ as the $\theta$ increased from $25^{\circ}$ to $40^{\circ}$ was found for wind speeds of $10 \mathrm{~m} / \mathrm{s}$, whereas almost no UW-DW asymmetry was observed. In Skrunes et al. (2016b), a preliminary study was presented based on four of the scenes in the UAVSAR time series described in the next section. In this paper, we extend the study presented in Skrunes et al. (2016b) to include the full time series and a larger set of parameters.

\section{Data Set}

The data set used in this analysis was collected during the NOrwegian Radar oil Spill Experiment (NORSE2015). The campaign was a collaboration between UiT The Arctic University of Norway, the Jet Propulsion Laboratory (JPL) / NASA, the Norwegian Meteorological Institute, and the Norwegian Clean Seas Association for Operating Companies (NOFO), and took place during NOFO's annual oil-on-water exercise at the abandoned Frigg field in the North Sea (around $59^{\circ} 59^{\prime} \mathrm{N}, 2^{\circ} 27^{\prime} \mathrm{E}$ ) on 10 June 2015. The experimental setup and collected data are described in the following subsections. 


\subsection{NORSE2015}

The objective of NORSE2015 was to collect SAR data over surface slicks with varying, known properties using different airborne and spaceborne sensors. Four different substances, three different emulsions and one plant oil, were released onto the open sea close in time. The emulsions were all based on Troll and Oseberg crude oils, but had varying oil volumetric fractions, i.e., 40\% (E40), 60\% (E60), and 80\% (E80). The plant oil (PO) was the Radiagreen ebo previously used for simulation of biogenic slicks (see Skrunes et al. (2014)). The behavior of the Radiagreen ebo has been found to differ somewhat from the expected characteristics of a natural biogenic slick (Jones et al., 2016a), and may not be a perfect proxy, but is still interesting for comparison to the mineral oils. The substances were released along a line approximately parallel to the flight (azimuth) direction of the SAR in order to keep the incidence angles of the different slicks roughly the same in each SAR image. To maximize the SNR, the releases were done close to the middle of the scenes. The volumes of the releases were $0.5 \mathrm{~m}^{3}$ for each of the emulsions and $0.2 \mathrm{~m}^{3}$ for the plant oil.

More detailed information about the NORSE2015 experimental setup, SAR data collection, and previous analyses can be found in Skrunes et al. (2016a); Brekke et al. (2016); Jones et al. (2016a); Espeseth et al. (2017, 2016); Jones et al. (2016b).

\subsection{Environmental Conditions}

Observations of meteorological and oceanographic conditions during the experiment were made from ships, buoys, drifters, and balloons. At the time of the four oil releases, the discharging ship measured wind speeds of $9-11 \mathrm{~m} / \mathrm{s}$ from a SW-W direction, a wave height of $2.5 \mathrm{~m}$, and a temperature of $9^{\circ} \mathrm{C}$. The wind conditions remained relatively high in the hours following the releases, with wind speeds between $9-12 \mathrm{~m} / \mathrm{s}$ and generally $10-12 \mathrm{~m} / \mathrm{s}$. The measured wind directions lay between $248^{\circ}-264^{\circ}$, with an average of $259^{\circ}$. Wave properties retrieved from satellite SAR data indicated that the direction of the waves was towards $129^{\circ}$. The wave direction is different from the in situ measured 
Table 2: Properties of the UAVSAR sensor (Fore et al., 2015).

\begin{tabular}{ll}
\hline \hline Frequency & $1.26 \mathrm{GHz}$ (L-band) \\
Mode & PolSAR \\
Look direction & Left \\
Polarization & Quad-polarization \\
Incidence angle & $19.5^{\circ}$ to $67.5^{\circ}$ \\
NESZ & $\sim-52 \mathrm{~dB}$ to $-35 \mathrm{~dB}$ \\
Resolution (range $\times$ azimuth) & $2.5 \mathrm{~m} \times 0.8 \mathrm{~m}$ \\
Scene size & $22 \mathrm{~km}$ swath \\
\hline \hline
\end{tabular}

local wind, and is likely an older wave system originating further out at sea (Skrunes et al., 2016a). The sea state was moderately rough, including some small breaking waves. Photos and further descriptions can be found in Jones et al. (2016a).

\subsection{UAVSAR Time Series}

The UAVSAR is an L-band SAR sensor, currently flown on a Gulfstream-III aircraft. It acquires high resolution quad-polarization data and has a very low noise floor (Fore et al., 2015). More information about the properties of the UAVSAR sensor can be found in Table 2 .

During NORSE2015 the UAVSAR had two flights, each lasting several hours, acquiring a time series of the evolving slicks consisting of 22 scenes in total, over a time period of almost eight hours. Data were collected from 05:32 - 08:53 (16 scenes) and from 11:45 - 13:18 (6 scenes) in flight 1 and 2, respectively. Hence, the ages of the slicks in the SAR imagery vary from about 45 minutes to 8.5 hours for the plant oil (released first), and from time of release to almost eight hours for the E80 (released last). A subscene of one of the earliest scenes (scene \#5) is shown in Fig. 1, with the four slicks and their estimated areas indicated. 


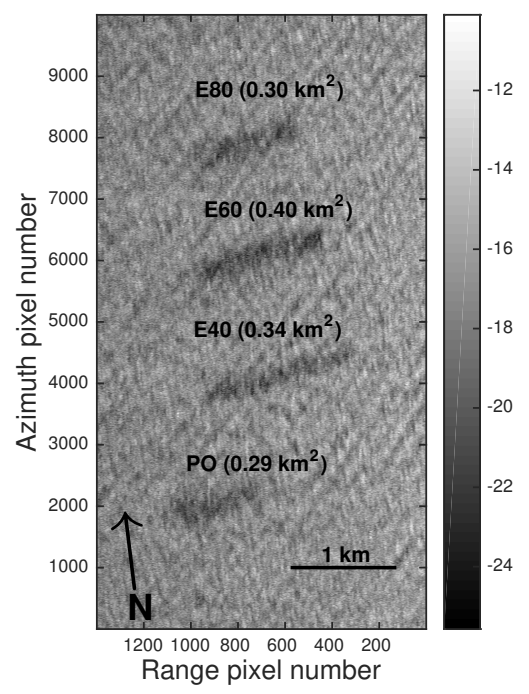

Figure 1: Intensity image $[\mathrm{dB}](\mathrm{VV})$ of scene \#5 with slick identities and areas indicated. UAVSAR data are courtesy of NASA/JPL-Caltech.

The UAVSAR acquired data on alternating ascending and descending passes along parallel lines, with a heading of $7^{\circ}$ (11 scenes) and $187^{\circ}$ (10 scenes), respectively. As the sensor is left-looking, the radar look direction was towards $277^{\circ}$ on ascending passes, and towards $97^{\circ}$ on descending passes. Hence, the sensor was looking close to upwind (exact upwind was on average $259^{\circ}$ ) for the ascending passes and close to downwind (exact downwind was on average $259^{\circ}$ $180^{\circ}=79^{\circ}$ ) for the descending passes. In addition, the last scene of flight 1 was collected with a heading of $142^{\circ}$ (i.e., look direction towards $52^{\circ}$ ). In this case, the look direction is also relatively close to downwind, but with a larger deviation than in the previous case. The radar flight and look direction relative to the swell and mean wind direction is shown in Fig. 2 for the three different flight lines, with the azimuth angles indicated. The scenes will hereafter be referred to as $\mathrm{UW}$ (flights with $\psi$ of $18^{\circ}$ ), $\mathrm{DW}_{1}$ (flights with $\psi$ of $198^{\circ}$ ), and $\mathrm{DW}_{2}$ (one flight with $\psi$ of $153^{\circ}$ ).

Each UAVSAR scene covers incidence angles of about $19.5^{\circ}-67.5^{\circ}$, but the 


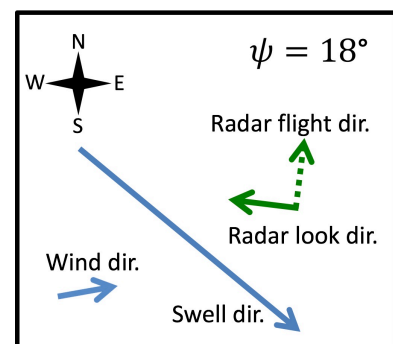

(a)

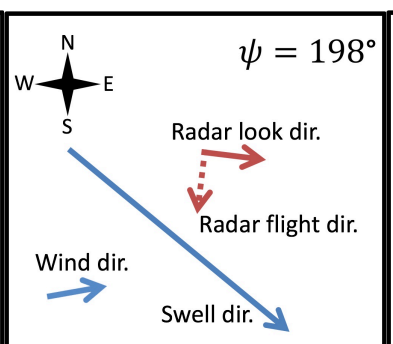

(b)

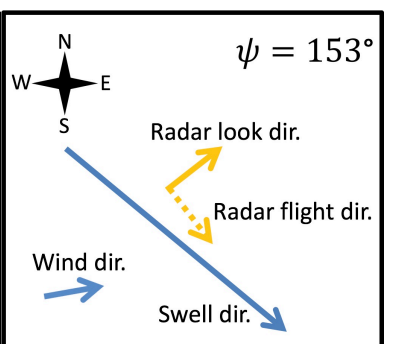

(c)

Figure 2: Overview of wind direction, swell direction, and radar flight and look directions for the three configurations, (a) UW, (b) $\mathrm{DW}_{1}$, and (c) $\mathrm{DW}_{2}$.

slicks span a much smaller $\theta$ range in each case. An overview of the scenes' imaging geometries, including the relative wind direction and incidence angle range of each slick, is given in Fig. 3. Each scene is shown in a separate color, with UW, $\mathrm{DW}_{1}$, and $\mathrm{DW}_{2}$ scenes represented by green colors, pink colors and orange, respectively. Scenes acquired early (late) in the time series are given light (dark) color shades. Note that the release of E80 was ongoing at the time of the acquisition of scene \#1, and is therefore not included for that particular scene in Fig. 3 or in the analysis to follow. In addition, some issues related to the calibration of scene \#6 prevents a direct comparison between this scene and the rest of the time series. Hence, we exclude scene \#6 from the analysis (and it is therefore presented in gray in Fig. 3).

\section{Results: Individual Polarization Channels}

In this paper, the effect of imaging geometry on the polarimetric UAVSAR data described in the previous section is investigated. The individual polarization channels are investigated in this section, whereas multipolarization parameters are discussed in Section 5.

Each UAVSAR scene covers incidence angles from about $19.5^{\circ}-67.5^{\circ}$, and the azimuth angle varies between scenes, allowing the dependency of the clean sea backscatter on these factors to be investigated. For each acquisition, a clean 


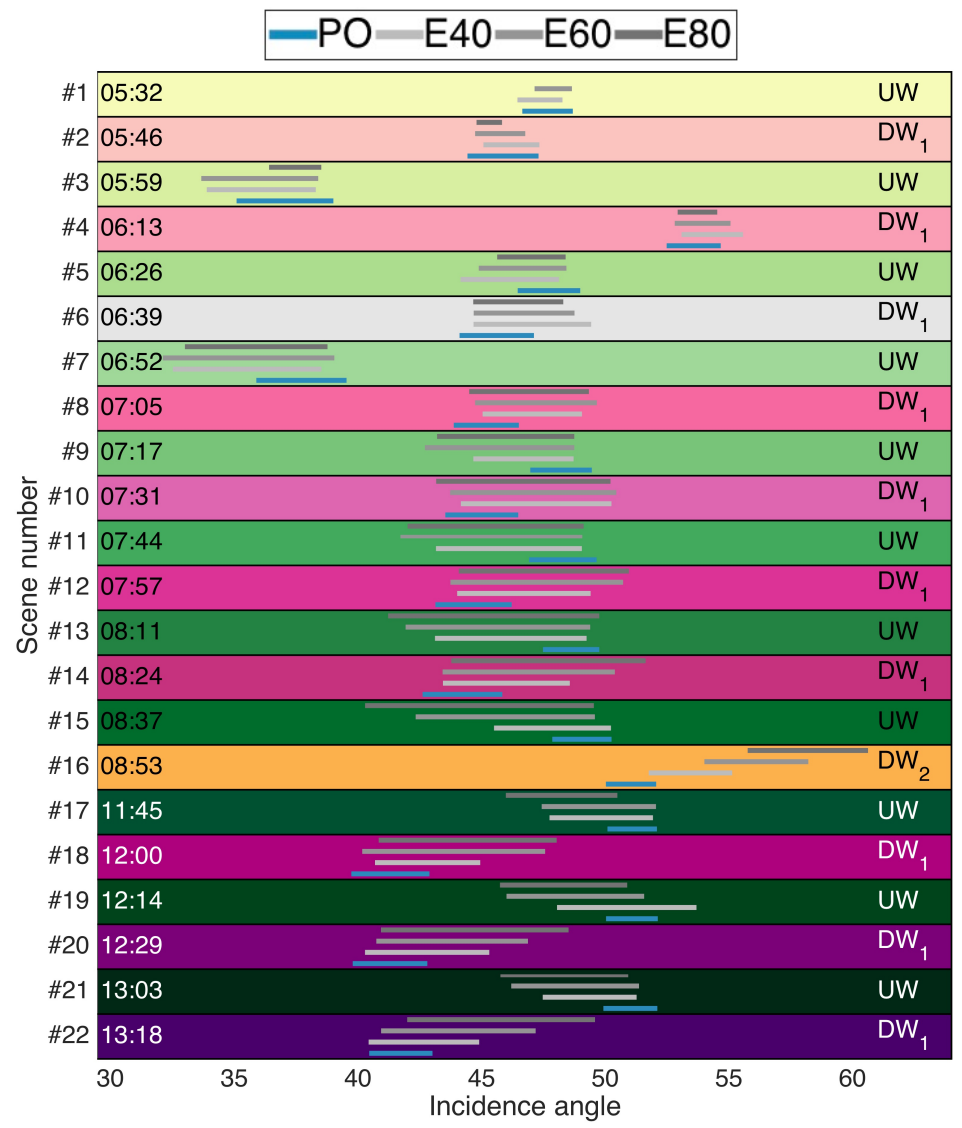

Figure 3: Overview of the UAVSAR time series, with the span of incidence angles covered by the slicks in each scene indicated. Relative wind directions and acquisition times are included on the right and left side, respectively. $\mathrm{UW}, \mathrm{DW}_{1}$, and $\mathrm{DW}_{2}$ scenes are represented by green colors, pink colors and orange, respectively, and change from light color shades early in the time series to darker shades towards the end. Scene \#6 is not included in the analysis and is therefore presented in gray. 
sea region covering the full scene in range direction and 1000 pixels in azimuth direction is selected north of the slick areas, avoiding ships and other bright targets. The selected area is first multilooked by a $15 \times 61$ (range $\times$ azimuth) pixels window, then averaged over azimuth to produce a profile of clean sea backscatter as a function of incidence angle. These profiles are plotted in Fig. 4 for the different polarization channels. The upper horizontal axis shows the Bragg wave number $k_{B}=2 \pi / \lambda_{B}$. Additional averaging over 200 pixels along the profile is applied to more clearly portray the large-scale variation. Each scene is plotted separately, in addition to the mean of all UW scenes (green dashed line) and the mean of all $\mathrm{DW}_{1}$ scenes (pink dashed line). The imbedded images in Fig. 4 are zoomed-out versions showing the backscatter levels relative to the noise floor, for both clean sea profiles and for the oil slick regions. For each slick, a vertical line is plotted between the 5th and the 95th percentiles of the backscatter values within the region (segmented using the extended polarimetric feature space method described in Espeseth et al. (2017)), with a star indicating the 50th percentile, and using the same color scale with respect to wind direction as for the clean sea dotted lines. No multilooking is applied prior to calculating these percentiles in order to show the characteristics of the actual measured values. Note that the main goal of the imbedded images in Fig. 4 is only to show the backscatter signal level compared to the noise floor. The characteristics of the oil slick backscatter will be discussed in more detail in Section 4.3.

Note that, for all three polarization channels in Fig. 4, some undulations can be seen along the profiles, especially pronounced at the higher incidence angles. In consultation with the UAVSAR processing group, it was determined that the ripples are probably not related to the backscattering, but rather to the calibration of the data because they fall mainly within the UAVSAR calibration accuracy of $0.7 \mathrm{~dB}$ (Fore et al., 2015). Hence, these variations will be ignored in the discussion of incidence angle variation in the following sections. We obtained calibration data from before and after the NORSE2015 campaign to better understand potential artifacts and limitations, and verified that the calibration accuracy reported in (Fore et al., 2015) was still valid for our study, 
with exceptions and limitations noted herein. Calibration, described in (Fore et al., 2015), is done using an array of corner reflectors, which are imaged at incidence angles up to $58^{\circ}$. Because we could not verify calibration accuracy for incidence angles above ca $60^{\circ}$, the results for these incidence angles are still included, but the area above this limit is indicated with a gray background in all the following plots, to indicate a higher uncertainty in these regions. Also, UAVSAR cross-polarization data collected over water has no cross-talk removal applied because the process does not work well over open water, and actually can introduce artifacts. Hence, for our study there is higher uncertainty in the absolute $\sigma^{0}$ values in cross-polarization channels than for the copolarization channels, as no cross-talk removal is carried out. The results for HV are still included in parts of the paper, but it should be noted that a higher uncertainty applies to these results.

\subsection{Backscatter Level vs. Sensor Noise Floor}

In Fig. 4, it is seen that clean sea backscatter in the VV channel is well above the NESZ for all $\theta$ and all scenes, whereas the HH backscatter approaches the noise floor at the very highest incidence angles. The HV channel has the lowest backscatter, which falls below the NESZ at $\theta$ around $65^{\circ}$. The clean sea backscatter profiles fall below the NESZ $+6 \mathrm{~dB}$ threshold used in Minchew et al. (2012) at $\theta \sim 58^{\circ}$ for $\mathrm{HV}$ and $\theta \sim 64^{\circ}$ in HH. For VV, the clean sea means are above this threshold for all incidence angles.

For the oil slicks, the 5 th percentile is well above the NESZ $+6 \mathrm{~dB}$ threshold for all slicks in the VV channel. In $\mathrm{HH}$, the 5 th percentile falls below the NESZ only for one slick (at $58^{\circ}$ ), whereas most slicks with $\theta>53^{\circ}$ have their 5 th percentiles below the $6 \mathrm{~dB}$ threshold. For the $\mathrm{HV}$ channel, most slicks located above $\theta \sim 48^{\circ}$ have their 5 th percentiles below the NESZ, and all slicks have their 5th percentiles below the $6 \mathrm{~dB}$ threshold. However, the 50th percentiles for $\mathrm{HV}$ still lie more than $6 \mathrm{~dB}$ above the NESZ for all slicks but one (located at the highest $\theta$ ). Only the slicks with their 50th percentiles above the $6 \mathrm{~dB}$ limit are included in the analyses presented in this paper. 


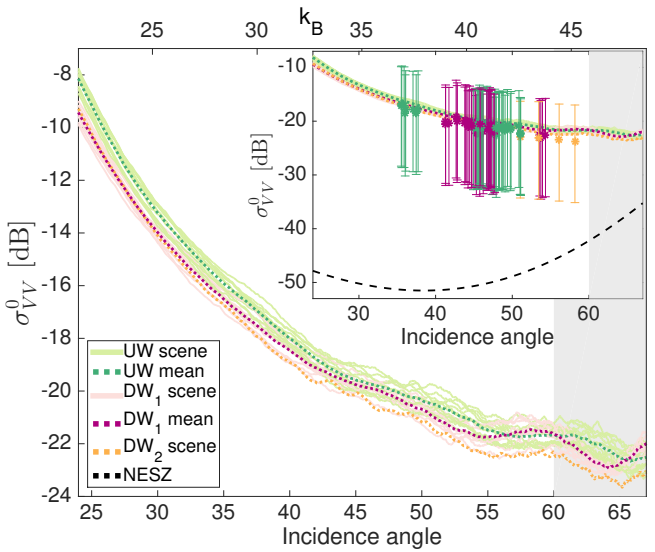

(a)

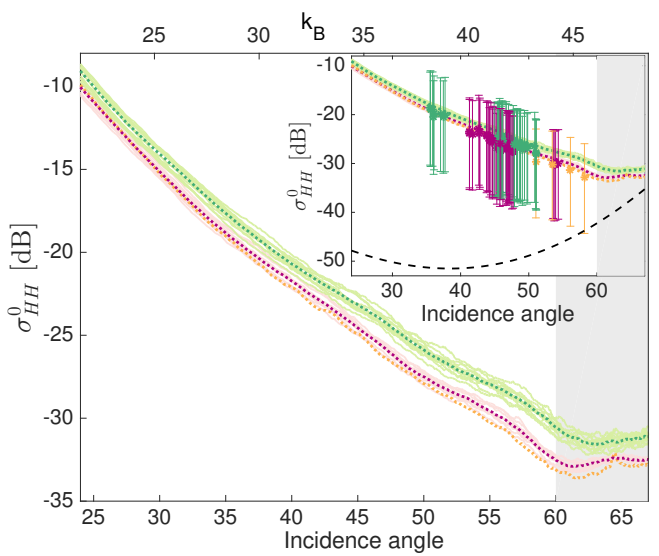

(b)

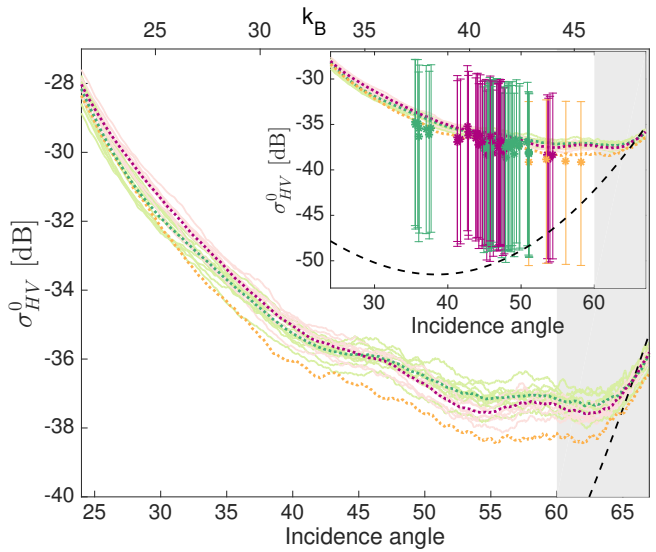

(c)

Figure 4: Clean sea backscatter as a function of $\theta$ (bottom x-axis) and $k_{B}$ (top x-axis) for (a) VV, (b) HH, and (c) HV. Single scene averages and the mean of all UW and $\mathrm{DW}_{1}$ scenes are shown. The imbedded images are zoomed-out versions showing the backscatter levels for both clean sea and oil slicks relative to the noise floor. Each oil slick is represented by a vertical line between the 5 th and 95 th percentiles of the single-look backscatter values. The area above $\theta$ $=60^{\circ}$ is gray shaded to indicate a higher calibration uncertainty. 
The low noise floor of the UAVSAR combined with the high wind conditions gives a high SNR for both the clean sea and slick covered regions in this data set, so that the sensor noise has a small effect on the results, especially in copolarization channels and for low to medium incidence angles.

\subsection{Clean Sea Backscatter}

Fig. 4 shows that the UAVSAR data set here investigated exhibits the same general backscatter characteristics observed previously and described in Section 2.1. For all polarization channels, the clean sea backscatter decreases as the incidence angle increases, with a more rapid decrease in $\mathrm{HH}$ than in the other two channels. The highest and lowest backscatter values are found in the $\mathrm{VV}$ and $\mathrm{HV}$ channels, respectively. At the highest incidence angles, the backscatter values flatten out and start to increase, especially in the HV channel. This is consistent with the measured $\sigma^{0}$ being a mixture of sensor noise and backscattered signal at high $\theta$ due to the proximity to the sensor noise floor, and has been observed previously (Minchew et al., 2012).

Fig. 4 shows that the variation in backscatter between the different scenes is relatively small, and the deviation between scenes with the same $\psi$ are mostly within $1 \mathrm{~dB}$. A dependency on the relative wind direction is observed. In $\mathrm{HH}$, the backscatter lies consistently higher in the UW scenes than in the DW scenes, and the difference increases with incidence angle up to about $60^{\circ}$. This is shown in more detail in Fig. 5, where the difference between the mean values of the UW scenes and $\mathrm{DW}_{1}$ scenes (i.e., the difference between the green and pink dashed lines in Fig. 4) is plotted. Note that, as the difference values are relatively small, the calibration related undulations along the profiles mentioned above has a clear effect on the plots. Hence, a linear fit to the data is included in Fig. 5. For $\mathrm{HH}$, the UW-DW difference is seen to increase from about $1 \mathrm{~dB}$ at low $\theta$ up to about $2 \mathrm{~dB}$ around $60^{\circ}$ (from ca $1.2 \mathrm{~dB}$ to $1.5 \mathrm{~dB}$ for the fitted line). Figs. 4 and 5 show that the UW scenes lie generally above the DW scenes also in VV for low to medium incidence angles, although the DW backscatter exceeds the UW backscatter in some areas due to the calibration-related undulations. However, 
the fitted line always lies above 0. For VV, the UW-DW difference decreases as the incidence angle increases, and at the lowest incidence angles, the difference between UW and DW backscatter is actually higher in VV than in $\mathrm{HH}$, with a cross-over at $\theta \sim 27^{\circ}$. However, this may be related to the calibration-related waves along profiles, and is not observed when looking at the fitted lines. As described in Section 2.1, the UW-DW asymmetry in the copolarization channels has been found to be mainly related to nonpolarized scattering, e.g., from wave breaking, which is more pronounced in UW than in DW. During the UAVSAR data collection, the wind speed was relatively high and some small breaking waves could be seen on the surface, which could result in the observed UWDW asymmetry. In Mouche et al. (2006), the variation with $\psi$ was found to be stronger in $\mathrm{HH}$ than in $\mathrm{VV}$, and to increase with incidence angles above $30^{\circ}$, which is in mainly in agreement with what we observe here. However, the decreasing difference in $\mathrm{VV}$ as $\theta$ increases was not observed in Mouche et al. (2006). It can be noted that for both $\mathrm{HH}$ and VV, the backscatter in the $\mathrm{DW}_{2}$ scene is similar to, or slightly lower than, the $\mathrm{DW}_{1}$ scenes, which may be due to the $\mathrm{DW}_{2}$ scene having a look direction further away $\left(\mathrm{DW}_{1}\right.$ at $18^{\circ} ; \mathrm{DW}_{2}$ at $\left.27^{\circ}\right)$ from directly downwind and closer to $\mathrm{CW}$, where a minimum in backscatter is expected.

From Fig. 4, it is seen that the HV channel has a somewhat different behavior than the copolarization channels, with less separation between UW and $\mathrm{DW}_{1}$ scenes. At incidence angles below ca $45^{\circ}$, the $\mathrm{DW}_{1}$ scenes have a slightly higher mean backscatter $(0-0.5 \mathrm{~dB})$ than the UW scenes, which is the opposite of the co-polarization channels. These findings are in agreement with the cross-polarization results described in Section 2.1. As the accuracy of the HV channels has a higher uncertainty than for copolarization channels (see beginning of Section 4), a more detailed comparison of HV data is not pursued here.

It should be noted that as we only have one scene with the $\mathrm{DW}_{2}$ geometry, the characteristics of this wind direction is more uncertain than that of UW and $\mathrm{DW}_{1}$. Hence, the following discussions will mainly compare the UW and $\mathrm{DW}_{1}$ scenes, which are acquired with exactly opposite look directions and in repeated 


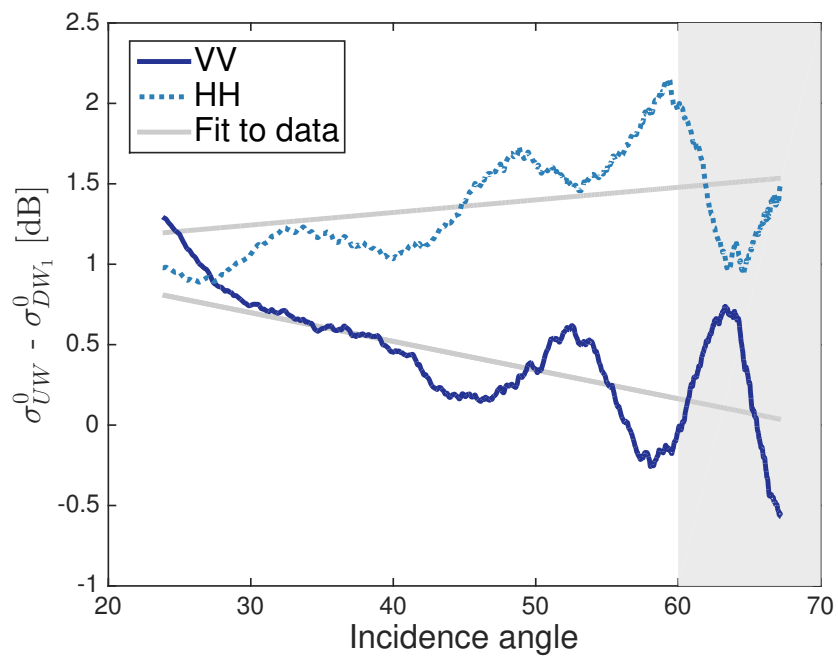

Figure 5: Difference between mean $\sigma^{0}[\mathrm{~dB}]$ of UW scenes and DW 1 scenes (i.e., the green and pink dashed lines in Fig. 4) for $\mathrm{HH}$ and VV channels. The gray lines are the linear polynomial curve fitting to the difference. The area above $\theta=60^{\circ}$ is gray shaded to indicate a higher calibration uncertainty.

passes.

\subsection{Oil Slicks Backscatter}

Profiles of the backscatter from oil covered regions cannot be obtained for the full range of incidence angles studied for the clean sea because the slicks cover only a small portion of the scene. That combined with the lower signal level from the slicks makes the dependency of oil slick backscatter on imaging geometry more difficult to evaluate than that of clean sea. The analysis is also complicated by the fact that the slicks are evolving over time, changing their properties (Espeseth et al., 2017). The general characteristics of the oil slick backscatter as a function of incidence angle and wind direction that can be obtained from the data is presented. Fig. 6 shows the characteristics of the backscatter from the oil covered regions, as well as the clean sea (only the mean per wind direction is here included). For each slick, a vertical gray line is plotted between the 5th and 95th percentiles and the 50th percentile is indicated by a 
$\psi$-dependent symbol in scene-specific colors as given by the legend (see also Fig. 3). No distinction between slick types are made in these plots. Note that the information plotted are similar to the imbedded images in Fig. 4, but here $15 \times 61$ pixels multilooking is applied prior to extracting the percentiles. After multilooking, all oil slicks have their 5th percentiles above the NESZ in all polarization channels.

The oil slick backscatter shows a similar variation with incidence angle as that of the clean sea, with values generally decreasing as $\theta$ increases, and with the most pronounced dependency in the HH channel. The variation with wind direction is more difficult to assess, and is complicated by the fact that the slicks in subsequent scenes are not necessarily at the same incidence angles, and the properties of the oil slicks can vary between acquisitions, especially early in the time series. Still, in many of the scenes, the slicks are located between $40^{\circ}-50^{\circ}$, and some comparison in terms of wind direction can be made. At these $\theta$, the slick regions show no clear difference between wind directions in $\sigma_{V V}^{0}$ and $\sigma_{H V}^{0}$, whereas $\sigma_{H H}^{0}$ has slightly higher values in UW compared to DW. These differences are the same as observed for clean sea. However, any difference due to wind direction is small compared to the within-slick variability. This is further discussed in the next section by looking at the damping ratio.

\subsection{Damping Ratio}

The preceding sections discuss how the backscatter values vary with imaging geometry. To evaluate how the damping of the signal within the oil slicks varies with these factors, we look at the damping ratio $\zeta$, i.e., the ratio between the mean backscatter value from a slick-free background sample, $\left\langle\left|S_{X Y \text {,sea }}\right|^{2}\right\rangle$, to the mean value of a slick-covered region, $\left\langle\left|S_{X Y \text {,oil }}\right|^{2}\right\rangle$ :

$$
\zeta=\frac{\left\langle\left|S_{X Y, \text { sea }}\right|^{2}\right\rangle}{\left\langle\left|S_{X Y, \text { oil }}\right|^{2}\right\rangle},
$$

535 where $X$ and $Y$ denotes transmit and receive polarization, respectively. The mean backscatter within each slick region is compared to a clean sea area selected at the exact same range position, only shifted in azimuth. As large areas 


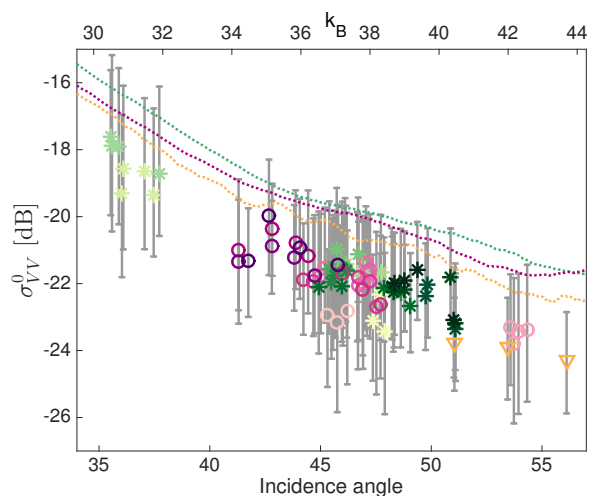

(a)

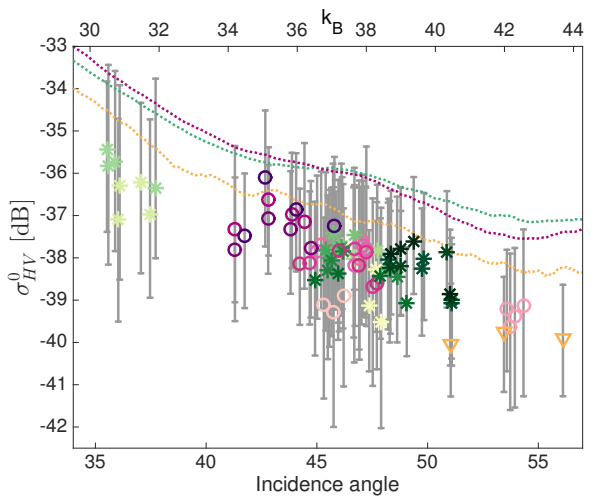

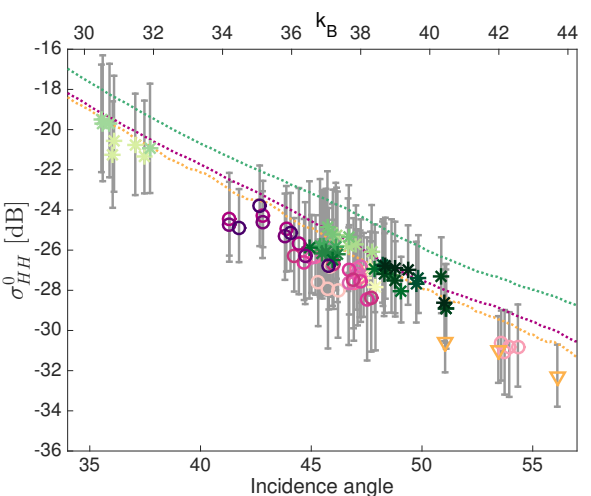

(b)

(c)

Figure 6: Backscatter as a function of $\theta$ (bottom x-axis) and $k_{B}$ (top x-axis) for (a) VV, (b) $\mathrm{HH}$, and (c) HV channels. Dashed lines are the mean ocean backscatter. For the oil slicks, vertical gray lines are plotted between the 5 th and 95th percentiles after multilooking, and the median is indicated by a $\psi$-dependent symbol in a scene-dependent color as given in the legend. 


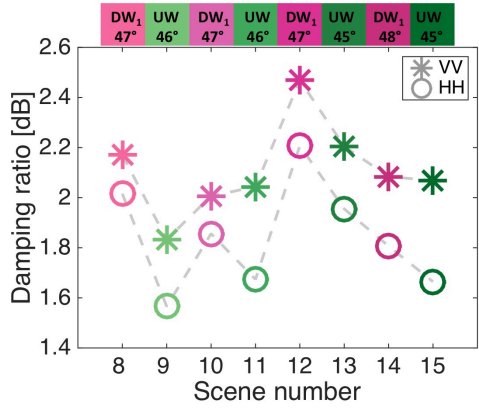

(a)

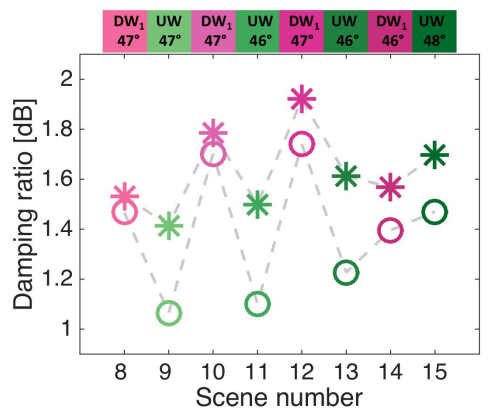

(c)

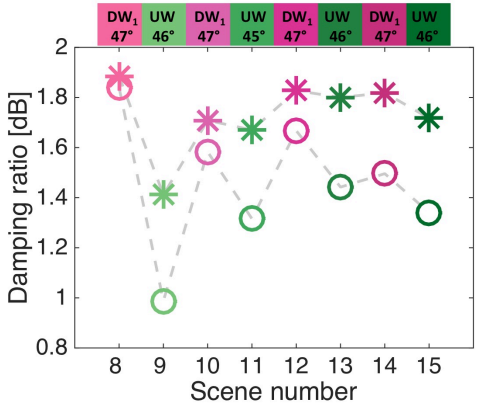

(b)

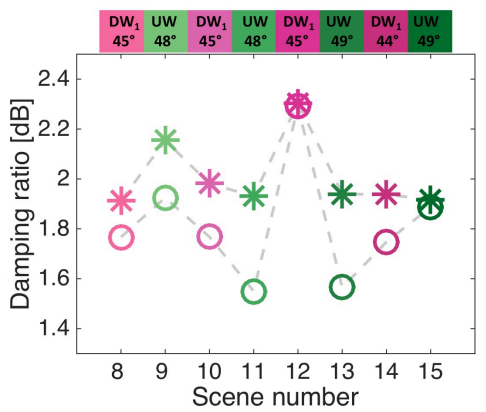

(d)

Figure 7: Damping ratios (in $\mathrm{dB}$ ) for the VV and HH intensities as function of scene number, for (a) E80, (b) E60, (c) E40, and (d) PO. Only scenes \#8 - \#15 are used for evaluating the $\psi$ sensitivity, as they are located after the initial $\zeta$ decrease following release and have nearly the same incidence angle for each slick. The imaging geometry for the slick is indicated along the top of each graph. 
of clean sea are available, a greater region is selected for the clean sea than for the oil slicks, but the relative distribution of pixels with respect to incidence angle is kept the same. The damping ratio for this data set was investigated in detail in Jones et al. (2016b), where the temporal evolution and variations between slick types and polarization channels were discussed. Hence, the current discussion only focuses on the variation of $\zeta$ with imaging geometry, in particular the wind direction.

Investigating the isolated effects of $\theta$ on the damping ratio is difficult as the only part of the time series where the incidence angle of subsequent scenes with the same wind direction varies significantly is the beginning of flight 1 (scenes \#1 - \#7), where the oils are relatively freshly released, and the temporal factor (spreading) is the main driver behind the changing $\zeta$ for the emulsions (see Jones et al. $(2016 \mathrm{~b}))$. Therefore, only the effect of $\psi$ on the damping ratio is evaluated here. Specifically, scenes \#8 - \#15 are used for this analysis, as these scenes are located after the initial $\zeta$ decrease, and have relatively stable incidence angles between acquisitions. The $\mathrm{HH}$ and $\mathrm{VV}$ damping ratios for scenes \#8-\#15 are plotted in Fig. 7, with the incidence angles at the slick centers given on top of each plot. The time span between the acquisition of scene \#8 and \#15 is ca 1.5 hours. In this period, the slick ages vary between $\sim 1.5$ hours and $\sim 4$ hours. The vertical axis varies between slicks, but the range in $\mathrm{dB}$ is constant. It was determined that computing the DR from a smaller random sample of data points within the slicks rather than using the full segmented slick regions has little effect on the DR value, resulting in very small variations around the values plotted in Fig. 7.

Fig. 7 shows some indications of a wind direction dependence, with higher $\zeta$ in DW scenes than in UW scenes in most cases, especially in the HH channel. This UW-DW difference is seen to some degree in all three emulsions for the $\mathrm{HH}$ channel, but is particularly pronounced in the E40 slick. If the backscatter from clean sea and from oil slicks had the same sensitivity to $\psi$, we would expect the $\zeta$ to be constant between scenes (assuming that the oil slick properties changes little over time in this part of the time series). Hence, the observed variation 
between UW and DW scenes indicates that the sensitivity to $\psi$ is different in slick-covered areas compared to in clean sea, particularly in the HH channel. According to the model described in Kudryavtsev et al. (2003, 2013); Mouche et al. (2006) (see Section 2.1), the backscatter can be written as the sum of a polarized Bragg scatter component and a non-polarized non-Bragg component related, e.g., to wave breaking, with the nonpolarized component found to be responsible for most of the UW-DW asymmetry. Previous studies have found the non-Bragg component to be less affected by the presence of oil films than the Bragg component, and to contribute relatively more with respect to the total backscatter signal within oil slicks compared to in clean sea (Kudryavtsev et al., 2013; Skrunes et al., 2015b). The larger contribution of the non-polarized component in oil slicks, together with the fact that this component is stronger in UW than in DW, can hence be the cause of the larger damping ratios here observed in DW. As the HH channel is more affected by this non-polarized component, it is reasonable that the $\psi$-dependency of $\zeta$ is most pronounced in this channel. Also, since the non-Bragg component contributes more to the total backscatter in the oil slicks compared to in clean sea, it can be expected that the oil covered areas are more affected by the $\psi$ than clean sea. This is here confirmed by comparing the backscatter in UW and DW of subsequent scenes (for scenes \#8 - \#15), which shows generally higher UW/DW ratios in oil covered areas than in clean sea. Results are shown in Table 3, where the ratio of the mean $\mathrm{HH}$ intensity between subsequent scenes are given for clean sea and mineral oil slicks. For each slick case and scene pair, the region (slick or sea) with highest UW/DW ratio is given in bold. For the majority of the cases, the slicks have higher UW/DW ratios than the corresponding clean sea region. Only the $\zeta$ for $\mathrm{HH}$ and VV are shown in Fig. 7 in order to simplify the plots, and as these channels are the most interesting and useful for the satellite based oil spill services. However, it can be mentioned that the HV damping ratio here has relatively high values, and mostly lie between those of the $\mathrm{HH}$ and VV, or in some cases even above the $\zeta$ for VV. The high $\zeta$ for HV could be partly related to depolarization effects due to presence of white caps on the sea surface caused 
Table 3: UW/DW ratios of the HH intensity for the emulsion slicks calculated between subsequent scenes. The region (slick or sea) with highest UW/DW ratio is presented in bold for each case.

\begin{tabular}{lllllllll}
\hline \hline \multirow{2}{*}{ Slick } & & $\frac{\# 9}{\# 8}$ & $\frac{\# 9}{\# 10}$ & $\frac{\# 11}{\# 10}$ & $\frac{\# 11}{\# 12}$ & $\frac{\# 13}{\# 12}$ & $\frac{\# 13}{\# 14}$ & $\frac{\# 15}{\# 14}$ \\
\hline \multirow{2}{*}{ E80 } & Sea & 1.60 & 1.63 & 1.53 & 1.65 & 1.67 & $\mathbf{1 . 8 3}$ & 1.83 \\
& Slick & $\mathbf{1 . 7 7}$ & $\mathbf{1 . 7 5}$ & $\mathbf{1 . 5 9}$ & $\mathbf{1 . 8 6}$ & $\mathbf{1 . 7 7}$ & 1.77 & $\mathbf{1 . 8 9}$ \\
\hline \multirow{2}{*}{ E60 } & Sea & 1.57 & 1.59 & 1.44 & 1.42 & 1.37 & 1.41 & 1.22 \\
& Slick & $\mathbf{1 . 9 1}$ & $\mathbf{1 . 8 3}$ & $\mathbf{1 . 5 3}$ & $\mathbf{1 . 5 4}$ & $\mathbf{1 . 4 5}$ & $\mathbf{1 . 4 3}$ & $\mathbf{1 . 2 7}$ \\
\hline \multirow{2}{*}{ E40 } & Sea & 1.41 & 1.31 & 1.24 & 1.25 & 1.17 & 1.17 & $\mathbf{0 . 9 8}$ \\
& Slick & $\mathbf{1 . 5 4}$ & $\mathbf{1 . 5 1}$ & $\mathbf{1 . 4 2}$ & $\mathbf{1 . 4 5}$ & $\mathbf{1 . 3 1}$ & $\mathbf{1 . 2 1}$ & 0.96 \\
\hline \hline
\end{tabular}

by the high wind. The between-scene variation in $\zeta$ for $\mathrm{HV}$ is more similar to that for VV than for HH, as expected from Figs. 4 and 5.

As mentioned in Section 2.2, the dependency of oil spill damping ratios on relative wind direction have been evaluated only in a few previous studies, which concluded that the damping was independent of the relative look direction. Many factors, including sensor system, oil properties, and wind conditions may cause differences between studies. As the variation in damping ratios here may be related to the non-polarized backscatter component, possibly from breaking waves, a similar UW-DW difference may not be observed in calmer wind conditions. However, this should be further investigated in the future, ideally keeping more factors constant between acquisitions to enable a more certain comparison.

\section{Results: Multipolarization Features}

The feature set introduced in Section 2.3 and listed in Table 1 is investigated to evaluate their sensitivity to imaging geometry. The results for clean sea areas are first discussed in Section 5.1, followed by a discussion of the oil slick regions in Section 5.2. 


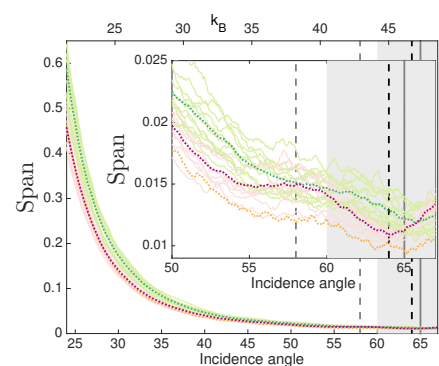

(a) Span

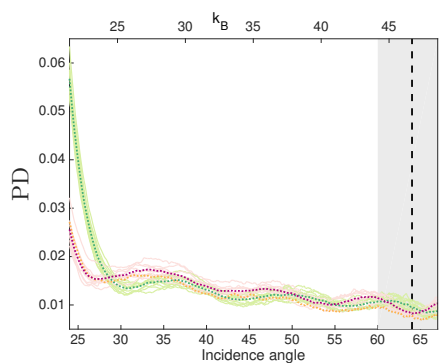

(d) Polarization difference, $P D$.

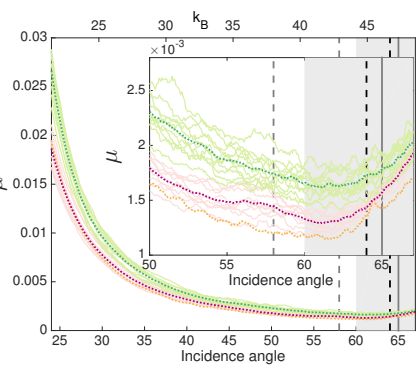

(b) Geometric intensity, $\mu$.

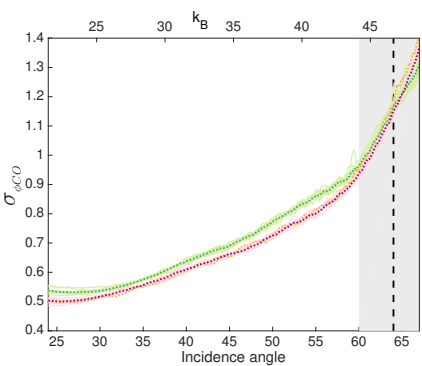

(e) Std. of copol. phase differ-

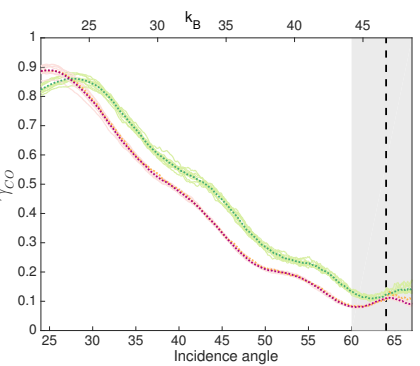

(c) Copol. power ratio, $\gamma_{C O}$.

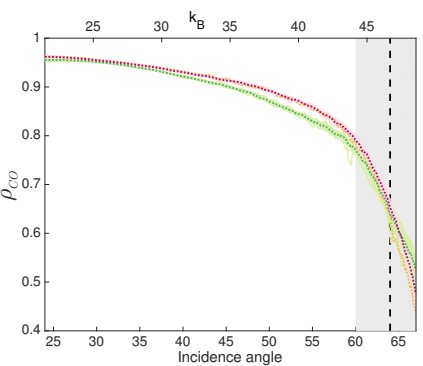

ence, $\sigma_{\phi C O}$

coefficient, $\rho_{C O}$.
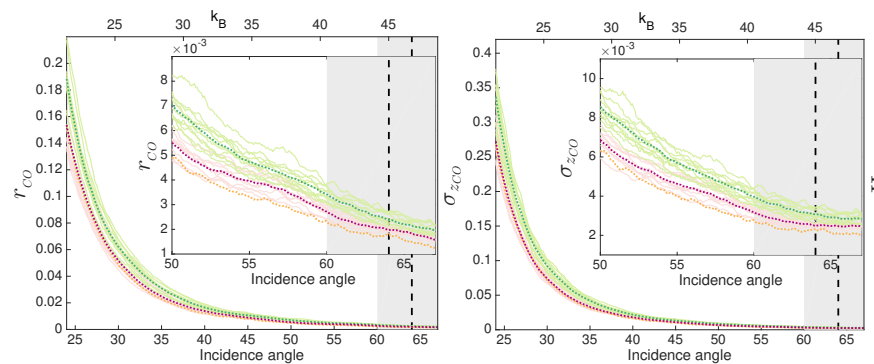

(g) Real part of copol. cross prod-(h) Std. of the copol. cross product, $r_{C O}$.

uct mag., $\sigma_{z C O}$.

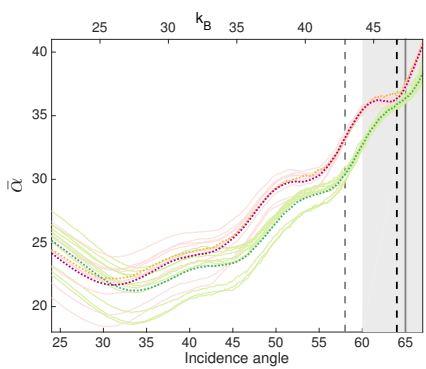

(j) Mean scattering angle, $\bar{\alpha}$.

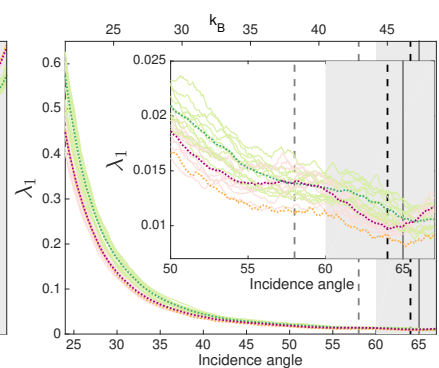

(k) Largest eigenvalue of $\mathbf{T}, \lambda_{1}$.

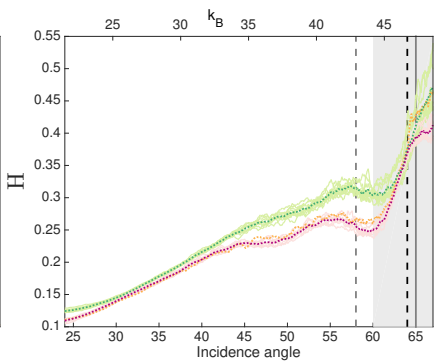

(i) Entropy, $H$.

Figure 8: Feature values over clean sea plotte $\$$ hs a function of $\theta$ (bottom x-axis) and $k_{B}$ (top $\mathrm{x}$-axis). Vertical solid (dashed) lines indicate the $\theta$ where the clean sea ocean backscatter falls below the NESZ (NESZ+6 dB) in HH (black) and HV (gray). The $\sigma^{0}$ in VV is always more than $6 \mathrm{~dB}$ above the noise floor. Inserts show close-ups of the profile tails at high $\theta$. The area above $\theta=60^{\circ}$ is gray shaded to indicate a higher calibration uncertainty. 


\subsection{Clean Sea}

Fig. 8 shows how the multipolarization feature values for clean sea vary with incidence angle and between scenes. All features are calculated using a $15 \times 61$ pixels window. The vertical solid (dashed) lines indicate the approximate $\theta$ where the clean sea backscatter in Fig. 4 falls below the NESZ (NESZ+6 dB limit used in Minchew et al. (2012)), respectively, for HH (black) and for HV (gray) where applicable. The area with $\theta>\sim 30^{\circ}$ is most relevant for satellite based remote sensing, but the $\theta$ range of $24^{\circ}-67^{\circ}$ is here shown for completeness. The area above $\theta=60^{\circ}$ is gray shaded to indicate a higher calibration uncertainty (see Section 4). For exponentially decreasing features, an insert of the tail region is included to more clearly show the behavior at the very highest incidence angles. It should be noted that the y-axis varies among the parameters and it can therefore be difficult to visually compare the dependency across features. Hence, in addition to the profile plots in Fig. 8, quantitative measures are applied to investigate and compare the features sensitivity to $\theta$ and $\psi$. The results are presented in Tables 4-6. In Table 4, the sensitivity to wind direction is quantified using the mean normalized difference (mnd) defined as:

$$
D_{m n d}=\frac{1}{N} \sum_{r=r_{1}}^{r_{1}+N-1} \frac{|U W(r)-D W(r)|}{0.5(U W(r)+D W(r))}
$$

where $U W(r)$ and $D W(r)$ is the mean clean sea profiles of the $\mathrm{UW}$ and $\mathrm{DW}_{1}$ scenes (i.e., the green and pink dashed lines in Fig. 8) respectively, in range position $r$, and $N$ is the number of pixels along range in the selected $\theta$ interval. The $D_{m n d}$ is calculated for the whole range of incidence angles, as well as for intervals of $\theta$, i.e., $30^{\circ}-40^{\circ}, 40^{\circ}-50^{\circ}$, and $50^{\circ}-60^{\circ}$. The intervals are included to avoid high/low $\theta$ effects (e.g., due to reduced SNR or other scattering types), and cover the most relevant incidence angles for spaceborne sensors. It is also of interest to evaluate whether there are parts of the range where the UW-DW difference is particularly high or low. In the case of future operational implementation, features with lower sensitivity to imaging geometry are preferable (given they have similar detection/characterization capabilities), to more easily 
develop general algorithms with a wide range of applicability. Hence, features with low UW-DW difference, i.e., low $D_{m n d}$, are preferred. In Table 4 , the five multipolarization parameters with the lowest $D_{m n d}$ are presented in bold for each column.

To quantify the dependency of the different parameters on incidence angle, the Spearmans correlation coefficient $\rho_{S}$ is first applied, and presented in Table 5 . The $\rho_{S}$ varies between -1 and 1 , with 0 indicating no correlation and \pm 1 indicating full correlation. Negative values indicate an inverse relation between feature values and $\theta$ (Corder and Foreman, 2009). For clean sea, the correlation between incidence angle and feature values is calculated for each scene for the data points with $30^{\circ} \leq \theta \leq 50^{\circ}$ to avoid effects at low and high $\theta$. The numbers presented in Table 5 are the median of $\rho_{S}$ for all UW scenes, for all DW $\mathrm{DW}_{1}$ scenes and the $\rho_{S}$ for the one $\mathrm{DW}_{2}$ scene available. The background colours represent different correlation categories, using a labelling system where $\left|\rho_{S}\right| \leq 0.35$ is considered weak (W) correlation, $0.36 \leq\left|\rho_{S}\right| \leq 0.67$ as moderate (M) correlation, $0.68 \leq\left|\rho_{S}\right| \leq 0.89$ as strong $(\mathrm{S})$ correlation, and $0.90 \leq\left|\rho_{S}\right|$ as very strong (VS) correlation (described in Taylor (1990) for the Pearson correlation coefficient). Results with $p$-values above 0.05 (i.e., not significant at significance level 0.05) are given in parentheses. Note that the results for the clean sea were significant in all cases except for one UW scene for the $\bar{\alpha}$ parameter, and that for the vast majority of the cases, the minimum and maximum values of $\rho_{S}$ were well within \pm 0.01 of the median value given in Table 5 . Only $P D$ had minimum and maximum values deviating as much as 0.09-0.17 from the median.

Although $\rho_{S}$ contains information about how correlated the features are with incidence angle, it doesn't provide information on how much the values vary across the range. Hence, the coefficient of variation $(C V)$ is also included to quantify the relative variation:

$$
C V=\frac{\sigma}{m}
$$

where $m$ and $\sigma$ is the mean and standard deviation of the feature values in the clean sea profile over a given range of incidence angles. The $C V$ is computed 
for each scene and for each feature, and the median of the results for all UW scenes and all $\mathrm{DW}_{1}$ scenes are presented in Table 6 . The $C V$ for the $\mathrm{DW}_{2}$ scene is not included, but the values are similar to those for $\mathrm{DW}_{1}$. It can be noted that the maximum and minimum values of $C V$ are generally well within the median (given in Table 6) \pm 0.03. Again, we are looking for features with low sensitivity to imaging geometry. As features can be highly correlated with $\theta$ while still having small changes in feature values as function of $\theta, C V$ is a better measure for the comparisons of relative changes in feature values, with low $C V$ s indicating low variation with $\theta$. In Table 6 , the five multipolarization parameters with the lowest $C V \mathrm{~s}$ are presented in bold for each case. To avoid the effects at the very high/low $\theta$, the selected incidence angle ranges are limited to $30^{\circ} \leq \theta \leq 60^{\circ}$. The quantitative measures presented in Tables $4-6$ are given for $\mathrm{HH}$ and $\mathrm{VV}$ intensities, in addition to the multipolarization features, for comparison.

A feature-by-feature discussion of the results presented in Fig. 8 and Tables $4-6$ is given in the following subsections.

\subsubsection{Span and Geometric Intensity $(\mu)$}

The Span and $\mu$ (Figs. 8(a)-8(b)) are both measures of the total intensity, and show a clear decrease with increasing incidence angle as expected. The values are larger for the UW scenes than for DW scenes, as observed for the copolarization backscatter in Fig. 4. A slight increase at the highest $\theta\left(\sim 62^{\circ}\right)$ is observed in $\mu$, but not clearly seen in the Span, indicating that $\mu$ may be slightly more affected by the proximity to the noise floor. Whereas the Span is just the sum of the intensities, the $\mu$ is based on all the elements of $\mathbf{T}$, including the phase information. Hence, $\mu$ may be more affected by the $\mathrm{HV}$ channel and its low SNR than the Span, where the copolarization channels dominate.

As noted in Section 4.2 and seen from Tables 4 and 6, the $\mathrm{HH}$ channel is clearly the polarization channel that is most sensitive to the imaging geometry. This sensitivity is somewhat diluted when extracting multipolarization features, where the measurements, and sensitivities, of the different channels are com- 
bined. Compared to the single polarization channels, Span, and $\mu$ mainly have $C V$ and $D_{m n d}$ values that lie between that of $\left\langle\left|S_{V V}\right|^{2}\right\rangle$ and $\left\langle\left|S_{H H}\right|^{2}\right\rangle$, but generally closer to the former. This indicates that Span and $\mu$ are less sensitive to imaging geometry, with more stable feature values under changing conditions, than the $\mathrm{HH}$ intensity. For $C V$, the $\mu$ even has values below that of $\left\langle\left|S_{V V}\right|^{2}\right\rangle$ for several cases.

Table 4 and Table 6 also show that $\mu$ has lower values of $C V$ than Span, i.e., less variation with $\theta$, particularly at low-medium $\theta$, whereas $\operatorname{Span}$ has lower $D_{m n d}$ than $\mu$, i.e., lower UW-DW difference, particularly at high $\theta$. Compared to the other multipolarization parameters, $\mu$ and Span have medium-high values of $C V$ and $D_{m n d}$. Table 5 shows that both features have $\rho_{S}=-1$, i.e., full correlation with $\theta$.

\subsubsection{Copolarization Power Ratio $\left(\gamma_{C O}\right)$}

The $\gamma_{C O}$ (Fig. 8(c)) has a clear dependency on both $\theta$ and wind direction, with values decreasing as the $\theta$ increases, and from UW to DW for $\theta$ between $\sim 30^{\circ}-60^{\circ}$. This is in accordance with the model and observations described in Mouche et al. $(2005,2006)$, which suggest that the increased $\gamma_{C O}$ in UW and at higher $\theta$ is due to a stronger contribution of non-polarized scattering here. At the very highest incidence angles, the $\gamma_{C O}$ values flatten out and increase, which is similar to the observations in Minchew et al. (2012), and could be related to the proximity to the noise floor.

One more characteristic of the $\gamma_{C O}$ profile in Fig. 8(c) that should be addressed is the wavy behavior along the profile, which is probably related to the calibration as discussed in Section 4.2. This behavior is seen in several features, but is especially pronounced in $\gamma_{C O}, P D$, and $\bar{\alpha}$. It can be noted that the undulations are not located at the exact same incidence angle for all features, because the oscillations also vary among the polarization channels (see Fig. 4). As the wavy behavior is assumed to be unrelated to variations in the backscatter, it is ignored in the following sections.

The quantitative measures in Tables $4-6$ show that $\gamma_{C O}$ has a $\rho_{S}$ of -1.0 , and 
Table 4: Mean normalized difference, $D_{m n d}$, between UW and $\mathrm{DW}_{1}$ scenes over clean sea. The five multipolarization parameters with the lowest $D_{m n d}$ are presented in bold for each column.

\begin{tabular}{lllll}
\hline \hline Feature & $\begin{array}{l}\mathbf{D}_{\text {mnd }} \\
\mathbf{2 4}^{\circ}-\mathbf{6 7}^{\circ}\end{array}$ & $\begin{array}{l}\mathbf{D}_{\text {mnd }} \\
\mathbf{3 0}^{\circ}-\mathbf{4 0}^{\circ}\end{array}$ & $\begin{array}{l}\mathbf{D}_{\text {mnd }} \\
\mathbf{4 0}^{\circ}-\mathbf{5 0}^{\circ}\end{array}$ & $\begin{array}{l}\mathbf{D}_{\text {mnd }} \\
\mathbf{5 0}^{\circ}-\mathbf{6 0}^{\circ}\end{array}$ \\
\hline$\left\langle\left|S_{V V}\right|^{2}\right\rangle$ & 0.09 & 0.14 & 0.06 & 0.07 \\
$\left\langle\left|S_{H H}\right|^{2}\right\rangle$ & 0.33 & 0.27 & 0.33 & 0.40 \\
\hline$S p a n$ & 0.12 & 0.19 & 0.13 & 0.09 \\
$\mu$ & 0.19 & 0.20 & 0.20 & 0.22 \\
$\gamma_{C O}$ & 0.27 & 0.13 & 0.27 & 0.37 \\
$P D$ & $\mathbf{0 . 1 0}$ & $\mathbf{0 . 1 1}$ & $\mathbf{0 . 1 0}$ & $\mathbf{0 . 0 8}$ \\
$\sigma_{\phi C O}$ & $\mathbf{0 . 0 4}$ & $\mathbf{0 . 0 4}$ & $\mathbf{0 . 0 5}$ & $\mathbf{0 . 0 6}$ \\
$\rho_{C O}$ & $\mathbf{0 . 0 2}$ & $\mathbf{0 . 0 1}$ & $\mathbf{0 . 0 2}$ & $\mathbf{0 . 0 3}$ \\
$r_{C O}$ & 0.20 & 0.20 & 0.19 & 0.21 \\
$\sigma_{z C O}$ & 0.20 & 0.22 & 0.20 & 0.21 \\
$H$ & $\mathbf{0 . 1 0}$ & $\mathbf{0 . 0 4}$ & $\mathbf{0 . 1 0}$ & 0.16 \\
$\bar{\alpha}$ & $\mathbf{0 . 0 6}$ & $\mathbf{0 . 0 4}$ & $\mathbf{0 . 0 8}$ & $\mathbf{0 . 0 7}$ \\
$\lambda_{1}$ & 0.11 & 0.19 & 0.12 & $\mathbf{0 . 0 7}$ \\
\hline \hline
\end{tabular}


relatively high values of $D_{m n d}$ and $C V$ compared to the other multipolarization features, especially at $\theta>40^{\circ}$. Hence, the $\gamma_{C O}$ values are more susceptible to changing $\theta$ and $\psi$ than the other features, which can be a disadvantage for operational use.

\subsubsection{Polarization Difference (PD)}

For $P D$ (Fig. 8(d)), a sharp decrease with increasing incidence angle is observed for $\theta<\mathrm{ca} .30^{\circ}$, after which a much slower decrease takes place. No clear effect on the general trend can be seen when approaching the sensor noise floor. There is a large degree of overlap between the different wind directions' clean sea profiles and no clear separation with respect to $\psi$ above $\sim 30^{\circ}$. The decreasing trend as $\theta$ increases and the lack of a clear wind direction dependence are in agreement with observations for C-band in Mouche et al. (2005, 2006). According to the model applied in those studies, the non-polarized component, which is the main component responsible for the UW-DW asymmetry, is removed by computing the difference between the copolarization channels.

Although we are here less concerned with the very lowest incidence angles, the clear separation between UW and DW data for $\theta<\sim 27^{\circ}$ should be commented on. In this region, the Bragg scatter may be less dominant, and other mechanisms, e.g., specular scattering may be more pronounced, and cause a larger difference between UW and DW that is not canceled out by looking at the polarization difference.

The $P D$ has $D_{m n d}$ values much lower than that of $\left\langle\left|S_{H H}\right|^{2}\right\rangle$, and close to that of $\left\langle\left|S_{V V}\right|^{2}\right\rangle$. Note that the calibration-related oscillations along the profile may cause an increase in the $D_{m n d}$ that is not physically based. The $C V$ for $P D$ is lower than or equal to that of $\left\langle\left|S_{V V}\right|^{2}\right\rangle$ and it can be concluded that much of the imaging geometry dependence of the individual channels are removed by looking at the $P D$. The lower values of $D_{m n d}$ and $C V$ for $P D$ compared to for $\gamma_{C O}$ also support the theory of a non-polarized additive component with a high sensitivity to imaging geometry, that cancels out in the $P D$. The $P D$ is also the only parameter with $\left|\rho_{S}\right|<0.96$, with values of $-0.64(\mathrm{UW}),-0.80\left(\mathrm{DW}_{1}\right)$ 
and -0.87 $\left(\mathrm{DW}_{2}\right)$, which are still considered moderate-strong correlation. It should be mentioned that the $\rho_{S}$ identifies monotonic functions between the two variables, and from Fig. $8(\mathrm{~d})$ it can be seen that for $P D$, the variation over $\theta$ is not monotonic. Hence, the resulting $\rho_{S}$ for $P D$ is less reliable. Note that the non-monotonic behavior of $P D$ may be due to the calibration related undulations previously mentioned rather than the change in $\theta$.

Overall, Tables 4 and 6 show that $P D$ has among the lowest values of $D_{m n d}$ and $C V$ of all the features, i.e., $P D$ is one of the best features in terms of feature value stability under varying imaging geometry.

\subsubsection{Copolarization Cross Product Parameters}

The real part of the copolarization cross product $\left(r_{C O}\right)$, the magnitude of the copolarization correlation coefficient $\left(\rho_{C O}\right)$, the standard deviation of the copolarization phase difference $\left(\sigma_{\phi C O}\right)$, and the standard deviation of the copolarization cross product magnitude $\left(\sigma_{z C O}\right)$ are all based on the copolarization cross product $S_{H H} S_{V V}^{*}$. The first three parameters describe the degree of correlation between $\mathrm{HH}$ and $\mathrm{VV}$, whereas $\sigma_{z C O}$ measures the variation in the correlation magnitude. The clean sea profiles in Figs. 8(e) - 8(g) show decreasing correlation between $\mathrm{HH}$ and $\mathrm{VV}$ as the incidence angle increases, i.e., decreasing values of $r_{C O}$ and $\rho_{C O}$ and increasing $\sigma_{\phi C O}$. Higher (lower) values of $\rho_{C O}$ $\left(\sigma_{\phi C O}\right)$ in DW than in UW indicates a slightly lower correlation between $\mathrm{HH}$ and $\mathrm{VV}$ in the former case, whereas higher $r_{C O}$ values in the UW indicates the opposite. It should be noted from Table 4 that $\rho_{C O}$ and $\sigma_{\phi C O}$ have very low values of $D_{m n d}$, whereas $r_{C O}$ shows a more significant difference between UW and DW data. Whereas $\rho_{C O}$ and $\sigma_{\phi C O}$ belong to feature category II (see Table 1), i.e., they are independent of the small-scale roughness, the $r_{C O}$ belongs to category I. Hence, the difference between these features in terms of sensitivity to wind direction can be related to the roughness.

The $\rho_{C O}$ and $\sigma_{\phi C O}$ also have little variation in feature values over $\theta$, as measured by the $C V$, compared to the other features, whereas the $r_{C O}$ has relatively high values and hence is more sensitive to both $\theta$ and $\psi$. Both $\rho_{C O}$ 


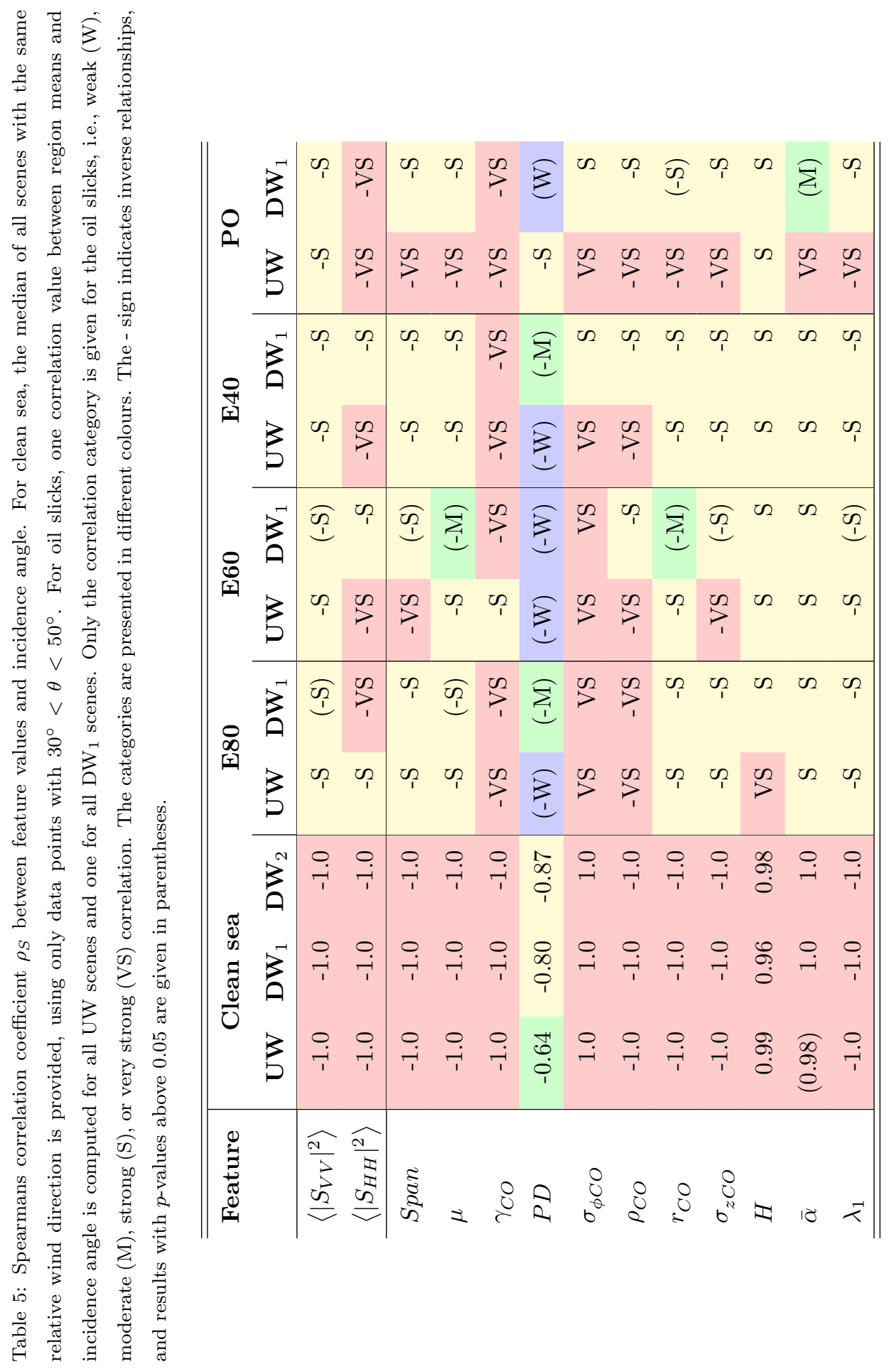


and $\sigma_{\phi C O}$ change behavior at the highest incidence angles, with a sharper decrease/increase above $\sim 60^{\circ}$, whereas $r_{C O}$ seems less affected by the high $\theta$ and the instrument noise. On the other hand, $r_{C O}$ has a steeper slope at the low incidence angles.

The $\sigma_{z C O}$ (Fig. 8(h)) shows decreasing values for increasing $\theta$, and from UW to DW, indicating a reduced variability in the cross correlation magnitude for high $\theta$ and DW conditions. Tables 4 and 6 show relatively large values of $C V$ and $D_{m n d}$, i.e., high dependency on imaging geometry, for $\sigma_{z C O}$ values. At the highest $\theta$, where the $\mathrm{HH}$ channel falls below the NESZ $+6 \mathrm{~dB}$ limit, the $\sigma_{z C O}$ profile flattens out.

Of the features related to the copolarization cross product, the category II parameters have among the lowest values of both $C V$ and $D_{m n d}$ of all the features, whereas the category II parameters have higher sensitivity to the imaging geometry. The $\rho_{S}$ indicates full correlation with $\theta$ for all the cross product parameters.

\subsection{5. $H / A / \bar{\alpha}$ Decomposition}

In the entropy $(H)$ (Fig. $8(\mathrm{i})$ ), a general increase with $\theta$ is observed. The values flatten out around $55^{\circ}-60^{\circ}$, before a rapid increase takes place above $60^{\circ}$. The behavior at high $\theta$ is probably related to the proximity to the NESZ and an increasing amount of noise mixed with the signal. A similar behavior was observed for $H$ in Minchew et al. (2012). The UW scenes have generally higher values than the DW scenes, and the difference increases with $\theta$ between $30^{\circ}$ and $60^{\circ}$. The higher entropy in UW, and the increasing difference with $\theta$, may be related to stronger contributions of the non-polarized scattering component in UW compared to DW, as discussed for $\gamma_{C O}$ and $P D$, and in Section 2.

In the most relevant part of the range, i.e., above $\theta \sim 30^{\circ}$, the mean scattering angle $(\bar{\alpha})$ generally increases with incidence angle, and lies a few degrees lower in UW than in DW. The differences are very small, and $\bar{\alpha}$ is found to be among the features with the lowest values of both $C V$ and $D_{m n d}$ in all cases. At the lowest incidence angles, a decrease in $\bar{\alpha}$ is observed as $\theta$ increases. The dif- 
ference in behavior for the lowest $\theta$ may again be related to different scattering properties, e.g., due to increased specular reflections. However, the differences are very small, i.e., only a few degrees. It can be noted that for $\theta<66^{\circ}$, all clean sea mean profiles have $H<0.5$ and $\bar{\alpha}$ below about $40^{\circ}$, indicating the presence of a dominating surface scattering mechanism, i.e., Bragg scattering, in all areas.

The clean sea profiles of $\lambda_{1}$ (Fig. $8(\mathrm{k})$ ) look very similar to those for Span, as expected when $\lambda_{1} \gg \lambda_{2}, \lambda_{3}$, resulting in Span $\approx \lambda_{1}$. The $\lambda_{2}$ and $\lambda_{3}$ are not included in this figure, but have values one to two orders of magnitude lower than $\lambda_{1}$. Hence, $\lambda_{1}$ (and Span) will be little affected by the noise. This is observed at the high $\theta$ in Fig. $8(\mathrm{k})$, where $\lambda_{1}$ seems unaffected by the proximity to the noise floor. This was also observed in Minchew et al. (2012).

Both $H$ and $\bar{\alpha}$ are among the features with the lowest values of $D_{m n d}$ and $C V$, i.e., among the best features in terms of feature value stability under varying imaging geometry. The $\lambda_{1}$ values vary more with imaging geometry, and produce very similar values as the Span. All three parameters have a very strong correlation with $\theta$.

The anisotropy $(A)$ is the last parameter in the $\mathrm{H} / \mathrm{A} / \bar{\alpha}$ decomposition and measures the relative importance of the second and third eigenvalues. However, as noted in Section 2.3, $A$ is only useful for high values of $H(H>0.7)$, when there is more than one scattering mechanism contributing to the signal. In this data set we have $H<0.5$ and $\lambda_{1} \gg \lambda_{2}, \lambda_{3}$, which means that the $A$ will be very contaminated by the noise. Hence, we choose to exclude the $A$ from the analysis as it does not contain any useful information.

\subsubsection{Feature Comparisons}

The results presented in Fig. 8 and Tables $4-6$ clearly shows that all parameters have some degree of sensitivity to the imaging geometry. For potential operational use, it is important to know how the applied parameters vary with these factors, and possibly identify features with less sensitivity to these conditions, i.e., low values of $C V$ and $D_{m n d}$. Comparing the $D_{m n d}$ in Table 4 for 
Table 6: Coefficient of variation, $C V$, for clean sea profiles within given ranges of incidence angles. The values presented are the medians of the $C V \mathrm{~s}$ for all scenes with the same relative wind direction. The five multipolarization parameters with the lowest $C V$ are presented in bold for each column.

\begin{tabular}{l|cl|ll|ll}
\hline \hline Feature & \multicolumn{2}{|c|}{$\mathbf{3 0}^{\circ}-\mathbf{4 0}^{\circ}$} & \multicolumn{2}{c}{$\mathbf{4 0}^{\circ}-\mathbf{5 0}^{\circ}$} & \multicolumn{2}{c}{$\mathbf{5 0}^{\circ}-\mathbf{6 0}^{\circ}$} \\
& $\mathbf{U W}$ & $\mathbf{D W}_{1}$ & $\mathbf{U W}$ & $\mathbf{D W}_{1}$ & $\mathbf{U W}$ & $\mathbf{D W}_{1}$ \\
\hline$\left\langle\left|S_{V V}\right|^{2}\right\rangle$ & 0.33 & 0.32 & 0.16 & 0.13 & 0.11 & 0.06 \\
$\left\langle\left|S_{H H}\right|^{2}\right\rangle$ & 0.48 & 0.48 & 0.35 & 0.39 & 0.29 & 0.33 \\
\hline$S p a n$ & 0.39 & 0.38 & 0.21 & 0.20 & 0.14 & 0.09 \\
$\mu$ & 0.27 & 0.26 & 0.14 & 0.16 & 0.10 & 0.08 \\
$\gamma_{C O}$ & 0.14 & 0.15 & 0.20 & 0.26 & 0.19 & 0.29 \\
$P D$ & $\mathbf{0 . 0 5}$ & $\mathbf{0 . 0 7}$ & $\mathbf{0 . 0 4}$ & $\mathbf{0 . 0 3}$ & $\mathbf{0 . 0 7}$ & $\mathbf{0 . 0 6}$ \\
$\sigma_{\phi C O}$ & $\mathbf{0 . 0 5}$ & $\mathbf{0 . 0 5}$ & $\mathbf{0 . 0 5}$ & $\mathbf{0 . 0 5}$ & $\mathbf{0 . 0 6}$ & $\mathbf{0 . 0 7}$ \\
$\rho_{C O}$ & $\mathbf{0 . 0 1}$ & $\mathbf{0 . 0 1}$ & $\mathbf{0 . 0 2}$ & $\mathbf{0 . 0 1}$ & $\mathbf{0 . 0 3}$ & $\mathbf{0 . 0 3}$ \\
$r_{C O}$ & 0.39 & 0.40 & 0.24 & 0.25 & 0.20 & 0.18 \\
$\sigma_{z C O}$ & 0.42 & 0.42 & 0.27 & 0.27 & 0.22 & 0.21 \\
$H$ & $\mathbf{0 . 1 1}$ & $\mathbf{0 . 1 1}$ & $\mathbf{0 . 0 7}$ & $\mathbf{0 . 0 4}$ & $\mathbf{0 . 0 5}$ & $\mathbf{0 . 0 4}$ \\
$\bar{\alpha}$ & $\mathbf{0 . 0 2}$ & $\mathbf{0 . 0 3}$ & $\mathbf{0 . 0 4}$ & $\mathbf{0 . 0 6}$ & $\mathbf{0 . 0 5}$ & $\mathbf{0 . 0 6}$ \\
$\lambda_{1}$ & 0.40 & 0.38 & 0.22 & 0.20 & 0.14 & 0.09 \\
\hline \hline
\end{tabular}


the different features, the minimum values are generally found in $\rho_{C O}, \sigma_{\phi C O}, \bar{\alpha}$, $P D$, and $H$, whereas $\gamma_{C O}$ has the maximum difference. The $D_{m n d}$ varies over range as seen from the last three columns in Table 4 , but the relative location of higher and lower differences varies among the features. For Span and $\lambda_{1}\left(\gamma_{C O}\right.$ and $H$ ) the $D_{m n d}$ decreases (increases) as $\theta$ increases, respectively. The other features have only small variations in $D_{m n d}$, i.e., relatively stable UW-DW difference over range. It should be noted that, as the UW-DW difference may be related to steep slopes and breaking waves, lower $D_{m n d}$ values may be observed in calmer wind conditions.

From columns 2-4 in Table 5 it is clear that most of the parameters have a very strong correlation with incidence angle, with $\left|\rho_{S}\right| \geq 0.96$ for all features except $P D$. However, a high correlation with $\theta$ may not necessarily cause large variations in feature values over range, as seen, e.g., in $\rho_{C O}$ and $\sigma_{\phi C O}$. The variation in feature values are measured by the $C V$, which shows some differences in feature variability over $\theta$, with the lowest values found in $\rho_{C O}, \bar{\alpha}, P D$, $\sigma_{\phi C O}$, and $H$. Note that these are the same five features that also produced the lowest values of $D_{m n d}$. The $C V$ changes across the range, but in different ways for the various features. No clear consistent differences with respect to wind direction are observed in the features' sensitivity to $\theta$ in either $C V$ or in $\rho_{S}$.

Fig. 8 shows that many features change behavior at the highest incidence angles. At least part of these changes can be due to the signal level approaching the noise floor (see Fig. 4), and the variations with $\theta$ cannot be evaluated without also taking the SNR into consideration. As the SNR is very high for the UAVSAR data, the signal is approaching the NESZ only at the very highest incidence angles. For satellite borne sensors, which are used operationally for oil spill detection, the SNR is often lower, and the signal can approach the NESZ at relatively low $\theta$. In that case, a corresponding plot of the feature values as seen in Fig. 8 may look different, with the effects here observed at high $\theta$ occurring at lower incidence angles. The effects of the proximity to the noise floor on the measurements and derived parameters can therefore be more important for the analysis of these data products. A similar investigation on the sensi- 
tivity to imaging geometry as presented in this paper is difficult for satellite sensors, as each scene only covers a few degrees of $\theta$ for quad-polarization SAR and acquisition of a larger set of scenes close in time, with varying observation geometry is not possible. However, we may expect a similar change in feature values as observed in Fig. 8 at the same SNR. Knowledge about these variations is important if multipolarization features are to be used more operationally for oil spill observation in the future. It can also be noted that, as oil slicks are low backscatter regions, the challenge of low SNR is even more important in these regions. Hence, increasing the knowledge on how a low SNR will modify multipolarization feature values is very important for a correct interpretation of these parameters (see discussions in, e.g., Minchew et al. (2012); Alpers et al. (2017)). Fig. 8 shows that the behavior at high $\theta$ (reduced SNR) varies between the features. In $\rho_{C O}, \sigma_{\phi C O}$, and $\bar{\alpha}$, the general trend at intermediate $\theta$ continues at the highest $\theta$, but with a larger slope, whereas in $\gamma_{C O}, H$, and $\mu$, a peak or trough and/or change in behavior seem to occur. In $\sigma_{z C O}$, the values flatten out when approaching the NESZ, whereas $r_{C O}$ and $P D$ seem unaffected by the high incidence angle and proximity to instrument noise. The Span and $\lambda_{1}$ are also little affected by the high incidence angle. A small increase may be present above $\theta \sim 65^{\circ}$, but this trend is difficult to distinguish from overall variations along the profiles. In the plots presented in Fig. 8, it can be seen that the changes in the feature values at high $\theta$ often occur at slightly lower incidence angles than the $6 \mathrm{~dB}$ threshold used in Minchew et al. (2012). In Fig. 4, the $\sigma_{H H}^{0}\left(\sigma_{H V}^{0}\right)$ seem to flatten out around $61^{\circ}-62^{\circ}\left(55^{\circ}\right)$, where the mean backscatter lines lie approximately $7-9 \mathrm{~dB}(8-9 \mathrm{~dB})$ above the noise floor, respectively. Hence, the measurements and derived multipolarization features may be affected by the proximity to the noise floor at even lower incidence angles/higher SNR than previously assumed. However, a separate study on this aspect will be carried out to thoroughly investigate the significance of the SNR. Comparing the categorization of features in Table 1 with the observations at high $\theta$, it seems like the parameters most affected by the proximity to the NESZ belong to category II, i.e., are independent of small-scale roughness, whereas 
the features less affected (or unaffected) by the NESZ are found in category I. Category II features, being ratios and having more terms involving the cross section, are more sensitive to the noise.

In Fig. 8, it can be seen that several features also have a different behavior at the very lowest incidence angles, below $\sim 30^{\circ}$. Category I parameters (Span, $\mu, P D, r_{C O}, \sigma_{z C O}$, and $\left.\lambda_{1}\right)$ have steeper slopes at the lowest $\theta$ than at intermediate and high $\theta$. The $\bar{\alpha}$ and $\gamma_{C O}$ have the visually most pronounced changes in behavior from low to intermediate incidence angles, with troughs or peaks around $30^{\circ}$. For $P D, \bar{\alpha}$, and $\gamma_{C O}$, a cross-over between UW and DW scenes are observed close to $\theta \sim 30^{\circ}$. Other scattering effects, e.g., specular scattering, may be important at the lowest incidence angles, possibly accounting for at least some of the differences in this part of the range.

Out of the three single-polarization intensities, HH clearly has the largest sensitivity to imaging geometry as seen from Fig. 4. The HH channel also has larger values of $D_{m n d}$ and $C V$ than the $\mathrm{VV}$ channel, over the whole $\theta$ range evaluated. The $D_{m n d}$ and $C V$ for the $\mathrm{HH}$ intensity is higher than for all multipolarization features, whereas VV has $D_{m n d}$ values closer to the features with the lowest $\psi$ sensitivity, and $C V$ values closer to the low-medium values found in the multipolarization parameters. Hence, some multipolarization features are less dependent on imaging geometry than single polarization channels, which could be an advantage in the case of future operational use.

The preceding sections have mainly focused on comparing the UW and $\mathrm{DW}_{1}$ scenes, which are acquired with exact opposite look directions, and in repeated passes. However, it can be noted from Fig. 8 that the feature profiles for the $\mathrm{DW}_{2}$ scene (yellow dashed line) is generally found close to, or among, the $\mathrm{DW}_{1}$ profiles in all features. The small differences between $\mathrm{DW}_{1}$ and $\mathrm{DW}_{2}$ feature values indicate that although the features depend on wind direction, there is also some robustness in the values around similar wind directions. 


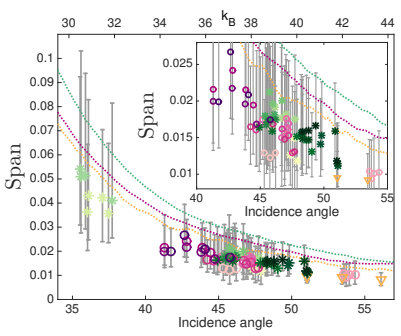

(a) Span.

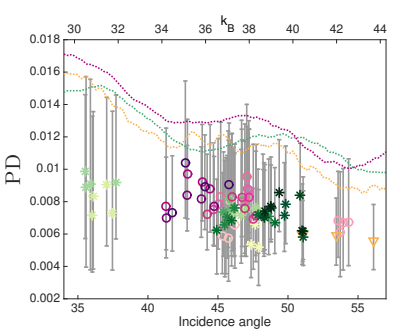

(d) Polarization difference, $P D$.

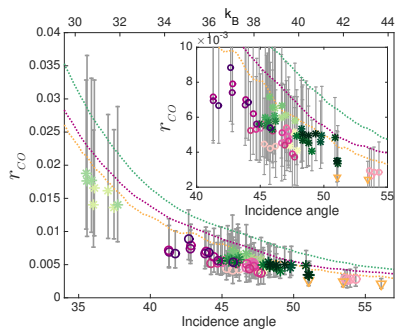

(g) Real part of copol product, $r_{C O}$.

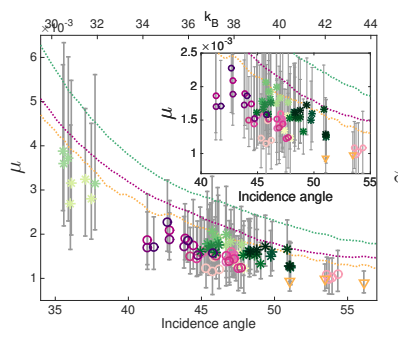

(b) Geometric intensity, $\mu$.

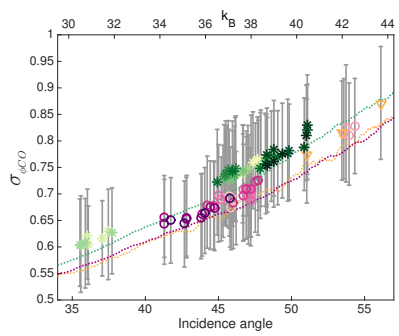

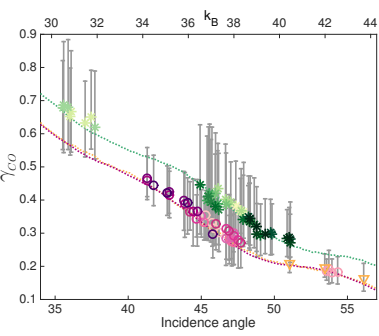

(c) Copol. power ratio, $\gamma_{C O}$.

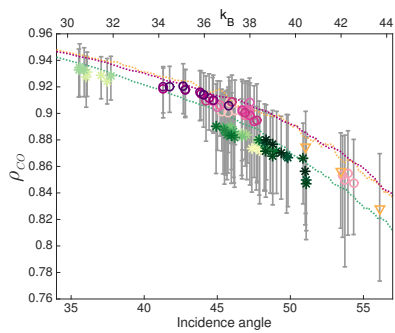

,(e) Std. of copol. phase differ-(f) Mag. of the copol. correlaence, $\sigma_{\phi C O}$.

tion coefficient, $\rho_{C O}$.

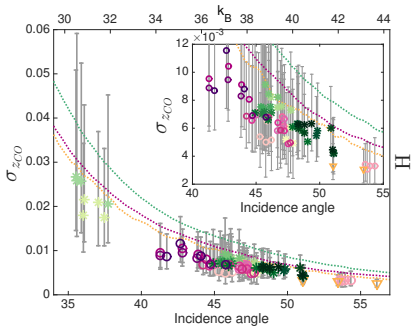

(h) Std. of the copol. cross product mag., $\sigma_{z C O}$.

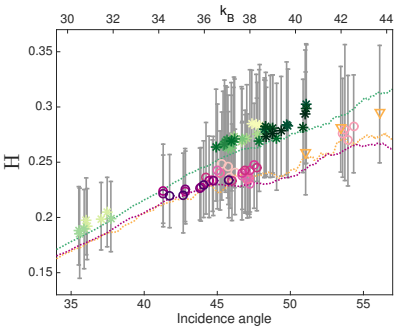

(i) Entropy, $H$.
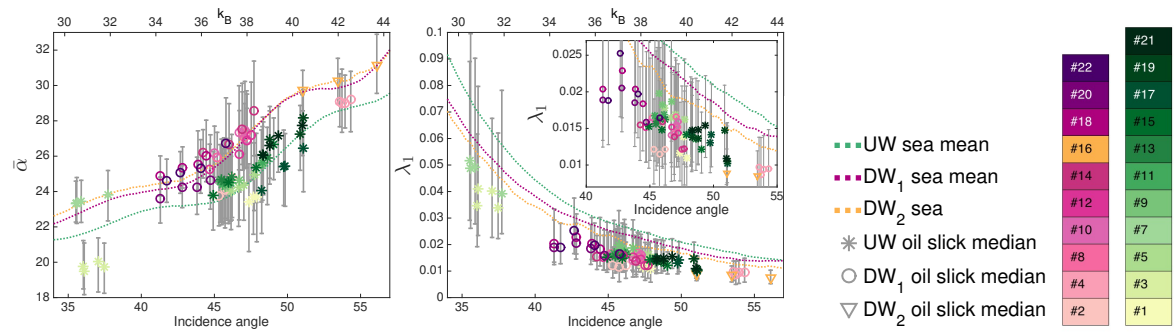

(j) Mean scattering angle, $\bar{\alpha}$. (k) Largest eigenvalue of $\mathbf{T}, \lambda_{1}$.

Figure 9: Feature values as a function of $\theta$ (bottom x-axis) and $k_{B}$ (top x-axis). For the oil slicks, vertical gray lines are plotted between the 5th and 95th percentiles, and the median is indicated by a $\psi$-dependent symbol with scene-dependent color as given in the legend. Inserts show close-ups of the $40^{\circ}<\theta<55^{\circ}$ region where necessary. 


\subsection{Oil Slicks}

The variation of multipolarization feature values with incidence angle and between scenes for the oil covered regions is plotted in Fig. 9. For each oil slick, a vertical gray line indicates the 5th to 95th percentiles of the feature values within the region, and the median is plotted with a $\psi$-dependent symbol in scene-specific colors as defined in the legend. No distinction is made among slick types. The clean sea mean for each relative wind direction is included for comparison. Inserts show close-ups of the $40^{\circ}<\theta<55^{\circ}$ region where necessary.

\subsubsection{Span and Geometric Intensity $(\mu)$}

The Span and $\mu$ values for the oil slicks (Figs. 9(a)-9(b)) decrease with increasing incidence angle in a similar way as observed for the clean sea. The within-region variability also decreases at higher $\theta$, as expected due to multiplicative speckle noise (Lee and Pottier, 2009). A clear wind direction dependency in slick values cannot be seen for Span, whereas in $\mu$, the median values seem to be slightly larger in UW than in DW, as observed for clean sea. However, the difference is small compared to the within-region variation. A clear decrease in Span and $\mu$ from clean sea to slicks is observed in all cases, mainly related to the damping of small-scale waves.

\subsubsection{Copolarization Power Ratio $\left(\gamma_{C O}\right)$}

The median values of $\gamma_{C O}$ for oil covered areas (Fig. 9(c)) closely follows the clean sea mean profiles, with the same decrease with increasing $\theta$ and from UW to DW. Hence, the $\gamma_{C O}$ is not a good parameter for oil spill detection in this data set. In fact, a poor oil-sea contrast is here observed in all parameters that are independent of small-scale roughness under the Bragg model, i.e., the ratiobased parameters in category II described in Section 2.3. This finding indicates that the dielectric properties are not sufficiently altered by the presence of slicks to be detected by SAR in this data set, and that the wave damping is the main factor (see discussion in Espeseth et al. (2017)). It should be noted that for thick slicks, this is likely to be different. 


\subsubsection{Polarization Difference (PD)}

The $P D$ (Fig. $9(\mathrm{~d})$ ) is seen to decrease from clean sea to oil slicks, reflecting the reduction in surface roughness. The slick-sea separability is good, with the 95th percentiles of the slick regions below the clean sea mean in many cases. As observed for clean sea, the oil slicks median values vary little over $\theta$, and no clear difference between UW and DW scenes can be seen.

\subsubsection{Copolarization Cross Product Parameters}

Figs. $9(\mathrm{e})-9(\mathrm{~g})$ show a decrease in real part of the copolarization cross product $\left(r_{C O}\right)$ and the magnitude of the copolarization correlation coefficient $\left(\rho_{C O}\right)$ and an increase in the standard deviation of the copolarization phase difference $\left(\sigma_{\phi C O}\right)$ in the oil slicks compared to in clean sea, all indicating a reduction in the HH-VV correlation. The change in correlation has previously been interpreted as a change in scattering mechanism (see, e.g., Nunziata et al. (2008)). Of the three parameters, $r_{C O}$ provides the best separation between slicks and sea, with several slicks having their 95th percentiles on or below the clean sea mean. This is probably because $r_{C O}$ belongs in category I, and is sensitive to the small-scale roughness, which is the main detection mechanism in place here for the thin slicks. The standard deviation of the copolarization cross product magnitude $\left(\sigma_{z C O}\right)($ Fig. $9(\mathrm{~h}))$ is also a category I parameter and shows a similar slick-sea difference as the $r_{C O}$. The values decrease from clean sea to oil-covered areas, indicating a reduced variability in the cross correlation magnitude in the oil slicks. For all the parameters in Figs. 9(e) - 9(h), the oil slicks show similar variation with incidence angle and wind direction, and among the features, as the clean sea.

\subsection{5. $H / A / \bar{\alpha}$ Decomposition}

The entropy $(H)$ and the mean scattering angle $(\bar{\alpha})$ (Figs. 9(i) - 9(j)) show similar variations with imaging geometry for the slicks as observed over clean sea, with values increasing with $\theta$ and from DW (UW) to UW (DW) for $H(\bar{\alpha})$. The oil covered areas have median $H$ values slightly above the clean sea mean, 
but slick-sea discriminability is poor, and the within-slick variations are large compared to the between-region differences. The oil-covered regions produce $\bar{\alpha}$ values (Fig. 9(j)) both higher and lower than the clean sea mean, but the values only differ by $1^{\circ}-2^{\circ}$. It can be noted that for all slicks included in Figs. 9(i)9(j), the 95 th percentiles have $H<0.36$ and $\bar{\alpha}<33^{\circ}$, indicating the presence of a dominating surface scattering mechanism, i.e., Bragg scattering. As for the clean sea, the results for $\lambda_{1}$ (Fig. 9(k)) look very similar to those for Span.

\subsubsection{Correlation with Incidence Angle}

The Spearmans correlation is also computed for oil slick regions, and presented in Table 5 . The correlation between the region means and the incidence angle at the center of the slicks is computed separately for UW and $\mathrm{DW}_{1}$ scenes and for each slick type. Due to the low number of data points (11 for UW and 9 for $\mathrm{DW}_{1}$ ), and the fact that the oil slick properties are evolving over time, the results for the slicks are more uncertain than for the clean sea. Therefore, only the correlation category is provided for the oil slicks.

Compared to the clean sea, the $\left|\rho_{S}\right|$ in the oil slicks is generally lower and varies more. However, the correlations are still relatively high, mainly within the strong and very strong correlation categories $\left(\left|\rho_{S}\right|\right.$ mostly above 0.7$)$. The multipolarization features showing the overall strongest correlation with $\theta$ for the oil slicks are $\gamma_{C O}, \sigma_{\phi C O}$, and $\rho_{C O}$. Overall, $P D$ is clearly the feature with lowest $\left|\rho_{S}\right|$ also for the slicks, with a weak correlation for the majority of the cases. Some variations in the correlation are observed between wind directions and slick types, but no consistent trends are clear. These findings were further investigated by computing the correlation coefficients for clean sea in a similar way as for the oil slicks, by using 9 (11) region means for $\mathrm{DW}_{1}$ (UW) scenes at the same incidence angles as the slicks, rather than the full profile lines (results not shown). In this case, the correlation for clean sea was reduced compared to when looking at the full profile, and there was a lot more variability in the values. Similar variations among "slick types" (i.e., clean sea regions located at the same range positions as the slicks and shifted in azimuth) and between wind 
directions were also observed. Hence, it is concluded that no clear variations in $\rho_{S}$ between wind directions or between slick types are found here.

\section{Conclusions}

Although the dependency of ocean backscatter on imaging geometry is well studied and described in the literature, few studies have looked at the effects on multipolarization parameters and on oil covered surfaces. This unique investigation was made possible by the capability of the airborne SAR to image the sea surface from different directions over a short time period, during which meteorological conditions and sea state varied little.

We find the characteristics of the clean sea backscatter to be in accordance with previous studies and scattering models, with decreasing $\sigma^{0}$ as the incidence angle increases, and a faster decrease in $\mathrm{HH}$ than in VV and HV. The $\mathrm{HH}$ channel also has the most pronounced variations with wind direction, with the highest backscatter in UW and the lowest in DW. Full $\theta$ profiles of the oil slick backscatter are not acquired, but the available measurements indicate a similar variation with $\theta$ and wind direction as for clean sea, but at a lower backscatter level. There are some indications of a higher damping ratio for the mineral oil slicks in DW scenes compared to UW scenes, particularly in the $\mathrm{HH}$ channel, which indicates a difference in the $\psi$ sensitivity between clean sea and oil slicks. The results suggest that the oil slicks have a slightly higher UW-DW difference than clean sea, which can be due to a higher contribution of nonpolarized non-Bragg scatter in slick-covered areas. Note that this may be more pronounced in high wind speeds, and the UW-DW difference in damping ratio here observed at 10-12 m/s may not be present in lower wind speeds. In even higher wind speeds, it could be even more pronounced. However, further studies are required to validate this. VV is already the preferred polarization channel for oil spill detection due to its higher oil-sea contrast and larger SNR. The reduced sensitivity to imaging geometry is another factor favoring this channel compared to HH. For the given sensor and conditions, the HV channel is also 
shown to be a good option for oil spill observation. On the other hand, HH may be useful as a means for understanding ocean and oil slick scattering differences under varying wind and wave conditions.

All the multipolarization features investigated here have a clear correlation with incidence angle, with $\left|\rho_{S}\right| \geq 0.96$ for the clean sea for all features except $P D$, which has $\rho_{S}$ between -0.64 and -0.87 , i.e., still moderate-strong correlation. The relative change in feature values with incidence angle are smallest for $\rho_{C O}, \bar{\alpha}, P D, \sigma_{\phi C O}$, and $H$. These five features also produce the lowest UW-DW differences. Note that features with high sensitivity to imaging geometry may still be useful for detection and/or characterization purposes. Under calmer wind conditions, the UW-DW differences may be lower than observed here. The feature values for the two different radar configurations with azimuth angles close to DW were overlapping, indicating some degree of robustness for data with similar look directions. Several multipolarization features have reduced sensitivity to imaging geometry compared to the individual polarization channels.

Although the investigated data set has a very high SNR, many of the multipolarization features show a difference in parameter values and behavior at the highest $\theta$, which can be related to the $\mathrm{HH}$ and/or HV signal approaching the noise floor. For this data set, we find that the features that seem to be least affected by the proximity to the NESZ are $r_{C O}, P D, \sigma_{z C O}$, Span, and $\lambda_{1}$, which depend upon the ocean wave spectra (category I parameters). In $\gamma_{C O}, H$, and $\mu$, a change in the general trend occurs at high $\theta$ (low SNR). There are indications that the measurements and derived parameters may be affected by the NESZ at even higher SNR than the NESZ + $6 \mathrm{~dB}$ limit previously proposed, i.e., closer to 7-9 dB above the noise floor. However, this aspect will be further investigated in the future.

Overall, the $P D$ stands out as a particularly interesting multipolarization parameter. In addition to a high oil-sea contrast, the $P D$ has an overall lower dependency on imaging geometry for $\theta$ greater than ca $30^{\circ}$ compared to most of the other features. These characteristics can be advantageous if implementing 
methods for use under a wide range of conditions. Other features producing low values of both $C V$ and UW-DW difference are $\rho_{C O}, \sigma_{\phi C O}$, and $\bar{\alpha}$. However, these parameters produce poor slick-sea contrasts for the slicks in our study, and seem to be at least in part more affected by the proximity to the sensor noise floor than the $P D$. For all the ratio-based parameters in category II, a poor oil-sea contrast is observed. This indicates that the dielectric properties of the surface are not sufficiently altered by the presence of the experimental slicks of this study to be detected by SAR. However, these results would probably be different for thicker oil slicks, for which the reduction in dielectric constant would also play a role. Hence, which parameters should be used may vary with type of slick and with the objective of the analysis, but in all cases, the features sensitivity to imaging geometry and SNR should be considered.

The results presented in this paper show that both the relative wind direction and the incidence angle (in combination with SNR) should be taken into account when developing methods based on multipolarization features. Studies like the one presented here may be further used to quantify the effects and possibly correct for them, or help establish how these properties should be used as input in a processing algorithm. However, similar analysis should be repeated for other sensors and imaging conditions. Other radar frequencies interact with surface waves of a different scale and have a different sensitivity to wind conditions, so conclusions drawn from our L-band study cannot be assumed to hold at X- or C-band. Hence, to obtain a more comprehensive understanding of sensitivity to wind direction, further studies should be done on the imaging geometry dependencies for other sensors, weather conditions, and types of slicks.

\section{Acknowledgement}

This study is funded by CIRFA (RCN grant no. 237906). The research described in this paper was carried out in part at the Jet Propulsion Laboratory, California Institute of Technology, under a contract with the National Aeronautics and Space Administration. The authors would like to thank NOFO for 
including our experiment in their exercise and for providing ground truth data, and scientists at the Norwegian Meteorological Institute for collecting metocean data. UAVSAR data are courtesy of NASA/JPL-Caltech. The authors thank Anthony Doulgeris for his segmentation code to generate the oil slick masks.

\section{References}

Alpers, W., B. Holt, and K. Zeng (2017), Oil spill detection by imaging radars: Challenges and pitfalls, Remote Sens. Environ., 201, 133-147.

Brekke, C., C. Jones, S. Skrunes, B. Holt, M. Espeseth, and T. Eltoft (2016), Cross-correlation between polarization channels in SAR imagery over oceanographic features, IEEE Geosci. Remote Sens. Lett., 13(7), 997-1001.

Brekke, C., S. Skrunes, and M. M. Espeseth (2017), Oil spill dispersion in fullpolarimetric and hybrid-polarity SAR, in Proc. IEEE Int. Geosci. Remote Sens. Symp., Forth Worth, Texas.

Cloude, S. R., and E. Pottier (1997), An entropy based classification scheme for land applications of polarimetric SAR, IEEE Trans. Geosci. Remote Sens., $35(1), 68-78$, doi:10.1109/36.551935.

Corder, G. W., and D. I. Foreman (2009), Nonparametric Statistics for NonStatisticians: A Step-by-Step Approach, John Wiley \& Sons, Inc.

Dagestad, K. F., J. Horstmann, A. Mouche, W. Perrie, H. Shen, B. Zhang, X. Li, F. Monaldo, W. Pichel, S. Lehner, M. Badger, C. B. Hasager, B. Furevik, R. C. Foster, S. Falchetti, M. J. Caruso, and P. Vachon (2012), Wind retrieval from synthetic aperture radar - an overview, in Proc. SeaSAR, pp. 213-234, Troms $\varnothing$, Norway.

Donelan, M. A. and W. J. Pierson (1987), Radar scattering and equilibrium ranges in wind-generated waves with application to scatterometry, J. Geophys. Res., 92(C5), $4971-5029$. 
Espeseth, M. M., S. Skrunes, C. Brekke, A.-B. Salberg, C. E. Jones, and B. Holt (2016), Oil spill characterization in the hybrid-polarity SAR domain using log-cumulants, in Proc. SPIE Remote Sens., Edinburgh, Scotland.

Espeseth, M. M., S. Skrunes, C. E. Jones, C. Brekke, B. Holt, and A. P. Doulgeris (2017), Analysis of evolving oil spills in full-polarimetric and hybridpolarity SAR, IEEE Trans. Geosci. Remote Sens., 55(7), 4190-4210.

Fore, A. G., B. D. Chapman, B. P. Hawkins, S. Hensley, C. E. Jones, T. R. Michel, and R. J. Muellerschoen (2015), UAVSAR polarimetric calibration, IEEE Trans. Geosci. Remote Sens., 53(6), 3481-3491.

Gade, M., W. Alpers, H. Hühnerfuss, H. Masuko, and T. Kobayashi (1998), Imaging of biogenic and anthropogenic ocean surface films by the multifrequency/multipolarization SIR-C/X-SAR, J. Geophys. Res., 103(C9), 18,85118,866 .

Hansen, M. W., V. Kudryavtsev, B. Chapron, C. Brekke, and J. A. Johannessen (2016), Wave breaking in slicks: Impacts on C-band quad-polarized SAR measurements, IEEE J. Sel. Topics Appl. Earth Observ. Remote Sens., 9(11), 4929-4940.

Holt, B. (2004), SAR imaging of the ocean surface, in Synthetic Aperture Radar Marine User's Manual, edited by C. Jackson and J. Apel, pp. 25-80, U.S. Department of Commerce, National Oceanic and Atmospheric Administration, Washington DC, USA.

Isoguchi, O., and M. Shimada (2009), An L-band ocean geophysical model function derived from PALSAR, IEEE Trans. Geosci. Remote Sens., 47(7), 19251936.

Jones, C. E., K.-F. Dagestad, Ø. Breivik, B. Holt, J. Röhrs, K. H. Christensen, M. Espeseth, C. Brekke, and S. Skrunes (2016a), Measurement and modeling of oil slick transport, J. Geophys. Res.: Oceans, 121, 7759-7775. 
Jones, C. E., M. M. Espeseth, B. Holt, C. Brekke, and S. Skrunes (2016b), Characterization and discrimination of evolving mineral and plant oil slicks based on L-band synthetic aperture radar (SAR), in Proc. of SPIE Remote Sens., Edinburgh, Scotland.

Kudryavtsev, V., D. Hauser, G. Caudal, and B. Chapron (2003), A semiempirical model of the normalized radar cross-section of the sea surface, 1. background model, J. Geophys. Res., 108(C3), FET 2-1-FET 2-24.

Kudryavtsev, V., B. Chapron, A. Myasoedov, F. Collard, and J. Johannessen (2013), On dual co-polarized SAR measurements of the ocean surface, IEEE Geosci. Remote Sens. Lett., 10(4), 761-765, doi:10.1109/LGRS.2012.2222341.

Latini, D., F. D. Frate, and C. E. Jones (2016), Multi-frequency and polarimetric quantitative analysis of the Gulf of Mexico oil spill event comparing different SAR systems, Remote Sens. Environ., 183, 26-42.

Lee, J.-S., and E. Pottier (2009), Polarimetric Radar Imaging, from basics to applications, CRC Press, Taylor and Francis Group, Boca Raton, USA.

Liu, P., X. Li, J. J. Qu, W. Wang, C. Zhao, and W. Pichel (2011), Oil spill detection with fully polarimetric UAVSAR data, Marine Pollution Bulletin, 62, 2611-2618.

Migliaccio, M., A. Gambardella, and M. Tranfaglia (2007), SAR polarimetry to observe oil spills, IEEE Trans. Geosci. Remote Sens., 45(2), 506 -511.

Migliaccio, M., F. Nunziata, and A. Gambardella (2009a), On the co-polarized phase difference for oil spill observation, Int. J. Remote Sens., 30(6), 15871602.

Migliaccio, M., A. Gambardella, F. Nunziata, M. Shimada, and O. Isoguchi (2009b), The PALSAR polarimetric mode for sea oil slick observation, IEEE Trans. Geosci. Remote Sens., 47(12), 4032 -4041. 
Migliaccio, M., F. Nunziata, A. Montuori, X. Li, and W. G. Pichel (2011a), A multifrequency polarimetric SAR processing chain to observe oil fields in the Gulf of Mexico, IEEE Trans. Geosci. Remote Sens., 49(12), 4729-4737.

Migliaccio, M., F. Nunziata, A. Montuori, and C. E. Brown (2011b), Marine added-value products using RADARSAT-2 fine quad-polarization, Can. J. Remote Sens., 37(5), 443-451.

Minchew, B., C. E. Jones, and B. Holt (2012), Polarimetric analysis of backscatter from the Deepwater Horizon oil spill using L-band synthetic aperture radar, IEEE Trans. Geosci. Remote Sens., 50(10), 3812-3830.

Mouche, A. A., D. Hauser, J.-F. Daloze, and C. G. rin (2005), Dual-polarization measurements at C-band over the ocean: Results from airborne radar observations and comparison with ENVISAT ASAR data, IEEE Trans. Geosci. Remote Sens., 43(4), 753-769.

Mouche, A. A., D. Hauser, and V. Kudryavtsev (2006), Radar scattering of the ocean surface and sea-roughness properties: A combined analysis from dualpolarizations airborne radar observations and models in c-band, J. Geophys. Res., 111(C9).

Nunziata, F., A. Gambardella, and M. Migliaccio (2008), On the Mueller scattering matrix for SAR sea oil slick observation, IEEE Geosci. Remote Sens. Lett., 5(4), 691-695.

Pinel, N., C. Bourlier, and I. Sergievskaya (2014), Two-dimensional radar backscattering modeling of oil slicks at sea based on the model of local balance: Validation of two asymptotic techniques for thick films, IEEE Trans. Geosci. Remote Sens., 52(5), 2326-2338, doi:10.1109/TGRS.2013.2259498.

Plant, W. J., V. Irisov (2017), A joint active/passive physical model for sea surface microwave signatures, J. Geophys. Res.: Oceans, 122, 3219-3239, 
Salberg, A., O. Rudjord, and A. Solberg (2014), Oil spill detection in hybridpolarimetric SAR images, IEEE Trans. Geosci. Remote Sens., 52(10), 65216533, doi:10.1109/TGRS.2013.2297193.

Singha, S., R. Ressel, D. Velotto, and S. Lehner (2016), A combination of traditional and polarimetric features for oil spill detection using TerraSAR-X, IEEE J. Sel. Topics Appl. Earth Observ. Remote Sens., 9(11), 4979-4990.

Skrunes, S., C. Brekke, and T. Eltoft (2014), Characterization of marine surface slicks by Radarsat-2 multipolarization features, IEEE Trans. Geosci. Remote Sens., 52(9), 5302-5319, doi:10.1109/TGRS.2013.2287916.

Skrunes, S., C. Brekke, and A. P. Doulgeris (2015a), Characterization of low backscatter ocean features in dual-copolarization SAR using log-cumulants, IEEE Geosci. Remote Sens. Lett., 12(4), 836-840.

Skrunes, S., C. Brekke, T. Eltoft, and V. Kudryavtsev (2015b), Comparing near coincident $\mathrm{C}$ - and X-band SAR acquisitions of marine oil spills, IEEE Trans. Geosci. Remote Sens., 53(4), 1958-1975.

Skrunes, S., C. Brekke, C. E. Jones, and B. Holt (2016a), A multisensor comparison of experimental oil spills in polarimetric SAR for high wind conditions, IEEE J. Sel. Topics Appl. Earth Observ. Remote Sens., 9(11), 4948-4961.

Skrunes, S., C. E. Jones, C. Brekke, B. Holt, and M. M. Espeseth (2016b), On the effect of imaging geometry on multipolarization SAR features for oil spill observation, in Proc. Living Planet Symposium, vol. 740, Prague, Czech Republic.

Taylor, R. (1990), Interpretation of the correlation coefficient: A basic review, J. Diagnostic Medical Sonography, 6, 35-39.

Thompson, D. R. (2004), Microwave scattering from the sea, in Synthetic Aperture Radar Marine User's Manual, edited by C. Jackson and J. Apel, chap. 4, pp. 117-138, U.S. Department of Commerce, National Oceanic and Atmospheric Administration, Washington DC, USA. 
Tian, W., Y. Shao, J. Yuan, S. Wang, and Y. Liu (2010), An experiment for oil spill recognition using RADARSAT-2 image, in Proc. IEEE Int. Geosci. Remote Sens. Symp., pp. 2761 -2764, Honolulu, USA.

Ulaby, F., R. K. Moore, and A. K. Fung (1986), Microwave remote sensing, active and passive, volume II; Radar remote sensing and emission theory, Artech House Inc., Norwood, USA.

Unal, C. M. H., P. Snoeij, and P. J. F. Swart (1991), The polarization-dependent relation between radar backscatter from the ocean surface and surface wind vector at frequencies between 1 and $18 \mathrm{GHz}$, IEEE Trans. Geosci. Remote Sens., 29(4), 621-626.

Vachon, P. W., F. M. Monaldo, B. Holt, and S. Lehner (2004), Ocean surface waves and spectra, in Synthetic Aperture Radar Marine User's Manual, edited by C. Jackson and J. Apel, pp. 139-169, U.S. Department of Commerce, National Oceanic and Atmospheric Administration, Washington DC, USA.

Valenzuela, G. R. (1978), Theories for the interaction of electromagnetic and oceanic waves - a review, Boundary-Layer Meteorology, 13(1-4), 61-85.

Velotto, D., M. Migliaccio, F. Nunziata, and S. Lehner (2011), Dual-polarized TerraSAR-X data for oil-spill observation, IEEE Trans. Geosci. Remote Sens., 49(12), 4751-4762.

Wismann, V., M. Gade, W. Alpers, and H. Hühnerfuss (1998), Radar signatures of marine mineral oil spills measured by an airborne multi-frequency radar, Int. J. Remote Sens., 19(18), 3607-3623.

Yueh, S., S. J. Dinardo, A. G. Fore, and F. K. Li (2010), Passive and active microwave observations and modeling of ocean surface winds, IEEE Trans. Geosci. Remote Sens., 48(8), 3087-3100.

Yueh, S., W. Tang, A. G. Fore, G. Neumann, A. Hayashi, A. Freedman, J. Chaubell, and G. S. E. Lagerloef (2013), L-band passive and active mi- 
crowave geophysical model functions of ocean surface winds and applications to Aquarius retrieval, IEEE Trans. Geosci. Remote Sens., 51(9), 4619-4632.

Yueh, S., W. Tang, A. G. Fore, A. Hayashi, Y. T. Song, and G. S. E. Lagerloef (2014), Aquarius geophysical model function and combined active passive algorithm for ocean surface salinity and wind retrieval, J. Geophys. Res.: Oceans, 119, 5360-5379.

Zhang, B., W. Perrie, X. Li, and W. G. Pichel (2011), Mapping sea surface oil slicks using RADARSAT-2 quad-polarization SAR image, Geophys. Res. Lett., $38(10)$.

Zhou, X., J. Chong, X. Yang, W. Li, and X. Guo (2017), Ocean surface wind retrieval using SMAP L-band SAR, IEEE J. Sel. Topics Appl. Earth Observ. Remote Sens., 10(1), 65-74. 